\title{
Research Article \\ Some Properties of the Complement of the Zero-Divisor Graph of a Commutative Ring
}

\author{
S. Visweswaran \\ Department of Mathematics, Saurashtra University, Rajkot 360 005, India \\ Correspondence should be addressed to S. Visweswaran, s_visweswaran2006@yahoo.co.in
}

Received 19 April 2011; Accepted 17 May 2011

Academic Editors: D. Anderson, V. Drensky, and D. Herbera

Copyright (C 2011 S. Visweswaran. This is an open access article distributed under the Creative Commons Attribution License, which permits unrestricted use, distribution, and reproduction in any medium, provided the original work is properly cited.

Let $R$ be a commutative ring with identity admitting at least two nonzero zero-divisors. Let $(\Gamma(R))^{c}$ denote the complement of the zero-divisor graph $\Gamma(R)$ of $R$. It is shown that if $(\Gamma(R))^{c}$ is connected, then its radius is equal to 2 and we also determine the center of $(\Gamma(R))^{c}$. It is proved that if $(\Gamma(R))^{c}$ is connected, then its girth is equal to 3 , and we also discuss about its girth in the case when $(\Gamma(R))^{c}$ is not connected. We discuss about the cliques in $(\Gamma(R))^{c}$.

\section{Introduction}

All rings considered in this note are nonzero commutative rings with identity. Unless otherwise specified, we consider rings $R$ such that $R$ admits at least two nonzero zerodivisors.

Let $R$ be a commutative ring with identity which is not an integral domain. Recall from [1] that the zero-divisor graph of $R$, denoted by $\Gamma(R)$, is the graph whose vertex set is the set of all nonzero zero-divisors of $R$ and distinct vertices $x, y$ are joined by an edge in this graph if and only if $x y=0$. Several researchers studied the zero-divisor graphs of commutative rings and proved several interesting and inspiring theorems in this area [1-14]. The research paper of Beck [9], the research paper of Anderson and Naseer [2], and the research paper of Anderson and Livingston [1] are first among several research papers that inspired a lot of work in the area of zero-divisor graphs. We denote by $Z(R)$ the set of all zero-divisors of $R$, and by $Z(R)^{*}$ the set of all nonzero zero-divisors of $R$.

Before we describe the results that are proved in this note, it is useful to recall the following definitions from [15]. Let $G=(V, E)$ be a connected graph. For any vertices $x, y$ of $G$ with $x \neq y, d(x, y)$ is the length of a shortest path in $G$ from $x$ to $y$ and $d(x, x)=0$ and the diameter of $G$ is defined as sup $\{d(x, y) \mid x$ and $y$ are vertices of $G\}$ and it is denoted by $\operatorname{diam}(G)$. 
For any $v \in V$, the eccentricity of $v$ denoted by $e(v)$ is defined as

$$
e(v)=\sup \{d(v, u) \mid u \in V\} .
$$

The set of vertices of $G$ with minimal eccentricity is called the center of the graph, and the minimum eccentric value is called the radius of $G$ and is denoted by $r(G)$.

It is known that for any commutative ring $R$ with identity which is not an integral domain, $\Gamma(R)$ is connected and $\operatorname{diam}(\Gamma(R)) \leq 3$ [1, Theorem 2.3]. In [13, Theorem 2.3], Redmond proved that for any Noetherian ring $R$ with identity which is not an integral domain, $r(\Gamma(R)) \leq 2$. Moreover, in Section 3 of [13] Redmond determined the center of $\Gamma(R)$ for any Artinian ring $R$. It is known that there are rings $R$ for which $r(\Gamma(R))=3[8$, Corollary 1.6]. In [14, Theorem 2.4], Karim Samei characterized vertices $x$ of $\Gamma(R)$ such that $e(x)=1$ where $R$ is a reduced ring. Furthermore, in the same theorem under some additional hypotheses on $R$, he described vertices $x$ of $\Gamma(R)$ such that $e(x)=2$ or 3 .

Let $G=(V, E)$ be a simple graph. Recall from [15, Definition, 1.1.13] that the complement of $G$ denoted by $G^{c}$ is defined by setting $V\left(G^{c}\right)=V$ and two distinct $u, v \in V$ are joined by an edge in $G^{c}$ if and only if there exists no edge in $G$ joining $u, v$.

It is useful to recall the following definitions from commutative ring theory before we proceed further. Let $I$ be an ideal of a ring $R, I \neq R$. A prime ideal $P$ of $R$ is said to be a maximal $N$-prime of $I$ in $R$ if $P$ is maximal with respect to the property of being contained in $Z_{R}(R / I)$ where $Z_{R}(R / I)=\{x \in R \mid x y \in I$ for some $x \in R \backslash I\}$ [16]. It is well known that if $\left\{P_{\alpha}\right\}_{\alpha \in \Lambda}$ is the set of all maximal $N$-primes of (0) in $R$, then

$$
Z(R)=\bigcup_{\alpha \in \Lambda} P_{\alpha}
$$

Let $I$ be an ideal of a ring $R$. A prime ideal $P$ of $R$ is said to be an associated prime of $I$ in the sense of Bourbaki if $P=\left(I:_{R} x\right)$ for some $x \in R$ [17]. In this case, we say that $P$ is a $B$-prime of $I$.

Let $R$ be a commutative ring with identity admitting at least two nonzero zerodivisors. In [18, Theorem 1.1], it was shown that $(\Gamma(R))^{c}$ is connected if and only if one of the following conditions holds.

(a) $R$ has exactly one maximal $N$-prime $P$ of $(0)$ such that $P$ is not a $B$-prime of $(0)$ in $R$.

(b) $R$ has exactly two maximal $N$-primes $P_{1}, P_{2}$ of (0) with $P_{1} \cap P_{2} \neq(0)$.

(c) $R$ has more than two maximal $N$-primes of (0).

Moreover, it was shown in [18] that if $(\Gamma(R))^{c}$ is connected, then $\operatorname{diam}\left((\Gamma(R))^{c}\right) \leq$ 3. In fact, it was shown in [18] that if either the condition (a) or the condition (c) of [18, Theorem 1.1] holds, then $\operatorname{diam}\left((\Gamma(R))^{c}\right)=2$. When the condition $(b)$ of [18, Theorem 1.1] holds, then it was shown in [18, Proposition 1.7] that $\operatorname{diam}\left((\Gamma(R))^{c}\right)=2$ if either $P_{1}$ is not a $B$-prime of (0) in $R$ or $P_{2}$ is not a $B$-prime of $(0)$ in $R$ and $\operatorname{diam}\left((\Gamma(R))^{c}\right)=3$ if both $P_{1}$ and $P_{2}$ are $B$-primes of $(0)$ in $R$.

For any set $A$, we denote by $|A|$, the cardinality of $A$. Whenever a set $A$ is a subset of a set $B$ and $A \neq B$, we denote it symbolically by $A \subset B$. If $X, Y$ are sets and if $X$ is not a subset of $Y$, we denote it symbolically $X \not \subset Y$. 
Let $R$ be a commutative ring with identity and let $\left|Z(R)^{*}\right| \geq 2$. If $(\Gamma(R))^{c}$ is connected, then we prove in Section 2 of this note that the radius of $(\Gamma(R))^{c}$ is equal to 2. Moreover, we observe that except in the case when condition (b) of [18, Theorem 1.1] holds, $\operatorname{diam}\left((\Gamma(R))^{c}\right)=r\left((\Gamma(R))^{c}\right)=2$ and so every vertex of $(\Gamma(R))^{c}$ is in the center of $(\Gamma(R))^{c}$. Furthermore, if condition (b) of [18, Theorem 1.1] holds and if (i) $R$ satisfies the further condition that either $P_{1}$ is not a $B$-prime of $(0)$ in $R$ or $P_{2}$ is not a $B$-prime of $(0)$ in $R$, then it is noted that each vertex of $(\Gamma(R))^{c}$ is in the center of $(\Gamma(R))^{c}$ and if (ii) both $P_{1}$ and $P_{2}$ are $B$-primes of $(0)$ in $R$, then it is verified that the center of $(\Gamma(R))^{c}=\left\{x \in Z(R)^{*} \mid\right.$ $P_{1} \neq\left((0):_{R} x\right)$ and $\left.P_{2} \neq\left((0):_{R} x\right)\right\}$.

Let $G=(V, E)$ be a graph. Recall from [15, Page 159] that the girth of $G$ denoted by $\operatorname{gr}(G)$ is defined as the length of a shortest cycle in $G$. If $G$ does not contain any cycle, then we set $\operatorname{gr}(G)=\infty[5]$.

Let $R$ be a commutative ring with identity which is not an integral domain. Several results are known about the girth of $\Gamma(R)[5,7]$. Indeed, it is known that for any commutative ring with identity which is not an integral domain, $\operatorname{gr}(\Gamma(R)) \leq 4$ if $\Gamma(R)$ contains a cycle [7, Proposition 2.2] and [12, 1.4]. In [5], Anderson and Mulay characterized commutative rings $R$ such that $\operatorname{gr}(\Gamma(R))=4$ [5, Theorem 2.2 and Theorem 2.3], and moreover, they characterized commutative rings $R$ such that $\operatorname{gr}(\Gamma(R))=\infty$ [5, Theorem 2.4 and Theorem 2.5].

In Section 3 of this paper we study about the girth of $(\Gamma(R))^{c}$ where $R$ is a commutative ring with identity satisfying the further condition that $\left|Z(R)^{*}\right| \geq 2$. If $(\Gamma(R))^{c}$ is connected, then it is shown that $\operatorname{gr}\left((\Gamma(R))^{c}\right)=3$.

Suppose that $R$ has only one maximal $N$-prime of $(0)$ and let it be $P$. If $P^{2} \neq(0)$ and if $(\Gamma(R))^{c}$ is not connected, then it is proved that $(\Gamma(R))^{c}$ contains a cycle if and only if $|P| \geq 5$ if and only if $\operatorname{gr}\left((\Gamma(R))^{c}\right)=3$.

Suppose that $R$ has exactly two maximal $N$-primes of (0) and let them be $P_{1}$ and $P_{2}$. If $(\Gamma(R))^{c}$ is not connected, then it is shown that $(\Gamma(R))^{c}$ contains a cycle if and only if $\left\{P_{1} \backslash P_{2} \mid \geq\right.$ 3 or $\left|P_{2} \backslash P_{1}\right| \geq 3$ if and only if $\operatorname{gr}\left((\Gamma(R))^{c}\right)=3$.

Let $G=(V, E)$ be a graph. Recall from [15, Definition 1.2.2] that a clique of $G$ is a complete subgraph of $G$. Moreover, it is useful to recall the definition of the clique number of $G$. Let $G=(V, E)$ be a simple graph. The clique number of $G$ denoted by $\omega(G)$ is defined as the largest integer $n \geq 1$ such that $G$ contains a clique on $n$ vertices [15, Definition, Page 185]. We set $\omega(G)=\infty$ if $G$ contains a clique on $n$ vertices for all $n \geq 1$.

Let $R$ be a commutative ring with identity which is not an integral domain. It is known that (i) $\omega(\Gamma(R))=\infty$ if and only if $\Gamma(R)$ contains an infinite clique, (ii) $\omega(\Gamma(R))<\infty$ if and only if $|\operatorname{nil}(R)|<\infty$ and $\operatorname{nil}(R)$ is a finite intersection of prime ideals of $R$ (i.e., the set of all minimal prime ideals of $R$ is finite) [9, Theorem 3.9] where nil $(R)$ is the nilradical of $R$. More interesting theorems were proved on $\omega(\Gamma(R))$ in [3, Section 3].

Let $R$ be a commutative ring with identity and let $\left|Z(R)^{*}\right| \geq 2$. In Section 4 of this paper we observe that if $R$ has at least two maximal $N$-primes of $(0)$, then $(\Gamma(R))^{c}$ does not contain any infinite clique if and only if $R$ is finite if and only if $\omega\left((\Gamma(R))^{c}\right)$ is finite. Let $n \geq 2$ and let $K_{1}, \ldots, K_{n}$ be finite fields. Let $R=K_{1} \times \cdots \times K_{n}$. We describe a method of determining $\omega\left((\Gamma(R))^{c}\right)$.

Let $R$ be a ring admitting exactly one maximal $N$-prime of (0). Let $P$ be the unique maximal $N$-prime of $(0)$ in $R$. Suppose that $(\Gamma(R))^{c}$ does not contain any infinite clique. Then it is verified in Section 4 that $P=\operatorname{nil}(R)$. Moreover, if $P^{2} \neq(0)$, then it is shown in Section 4 that $P$ is a $B$-prime of $(0)$ in $R$ and furthermore, $R / P$ is a finite field and $R$ satisfies d. c. c. on principal ideals. As a corollary, we deduce that if $R$ is either a Noetherian ring or a chained ring, then $(\Gamma(R))^{c}$ does not contain any infinite clique if and only if $R$ is finite. Let $R$ be a 
finite chained ring with $P$ as its unique maximal $N$-prime of $(0)$. If $P^{2} \neq(0)$, then we provide a formula for computing $\omega\left((\Gamma(R))^{c}\right)$.

Let $R$ be a ring with $\left|Z(R)^{*}\right| \geq 2$. Suppose that $R$ has only one maximal $N$-prime of (0) and let it be $P$. If $\omega\left((\Gamma(R))^{c}\right)$ is finite, then it is shown in Section 4 that $P$ is nilpotent.

We end this note with an example of an infinite ring $R$ such that $R$ has exactly one maximal $N$-prime of $(0)$ with the property that $\omega\left((\Gamma(R))^{c}\right)=3$.

\section{The Radius of $(\Gamma(R))^{c}$}

Let $R$ be a ring with $\left|Z(R)^{*}\right| \geq 2$. We assume that $(\Gamma(R))^{c}$ is connected. The aim of this section is to show that the radius of $(\Gamma(R))^{c}$ is equal to 2 . We make use of the following lemmas for proving the result that $r\left((\Gamma(R))^{c}\right)=2$.

Lemma 2.1. Let $G=(V, E)$ be a simple and connected graph with $|V| \geq 2$ satisfying the further condition that $G^{c}$ is also connected. If $x$ is any element of $V$, then $e(x) \geq 2$ in $G^{c}$.

Proof. Let $x \in V$. Since $|V| \geq 2$ and $G$ is connected, it follows that there exists $y \in V$ such that $x$ is adjacent to $y$ in $G$. Thus $x$ is not adjacent to $y$ in $G^{c}$. Hence $d(x, y) \geq 2$ in $G^{c}$. This proves that $e(x) \geq 2$ in $G^{c}$.

The following lemma establishes some necessary conditions on $R$ in order that there exist $x, y \in Z(R)^{*}$ such that $d(x, y)=3$ in $(\Gamma(R))^{c}$.

Lemma 2.2. Let $R$ be a ring with $\left|Z(R)^{*}\right| \geq 2$. Suppose that $(\Gamma(R))^{c}$ is connected. If there exist $x, y \in Z(R)^{*}$ with $d(x, y)=3$ in $(\Gamma(R))^{c}$, then $R$ has exactly two maximal $N$-primes of $(0)$ and moreover, both of them are B-primes of $(0)$ in $R$. Indeed, if $P$ and $Q$ are the maximal $N$-primes of $(0)$ in $R$, then $\left.\{P, Q\}=\left\{(0):_{R} x\right),\left((0):_{R} y\right)\right\}$.

Proof. Since $d(x, y)=3$ in $(\Gamma(R))^{c}$, it follows that $x y=0$ and for any $z \in Z(R)^{*}$ with $z \notin$ $\{x, y\}$, either $z x=0$ or $z y=0$. Hence we obtain that $z \in\left((0):_{R} x\right) \cup\left((0):_{R} y\right)$. Since $x y=0$, it is clear that $\{0, x, y\} \subseteq\left((0):_{R} x\right) \cup\left((0):_{R} y\right)$. Thus we have $Z(R)=\left((0):_{R} x\right) \cup\left((0):_{R} y\right)$. Let $\left\{P_{\alpha}\right\}_{\alpha \in \Lambda}$ be the set of all maximal $N$-primes of (0) in $R$. It is well known that $Z(R)=\bigcup_{\alpha \in \Lambda} P_{\alpha}$, and hence we obtain that $\bigcup_{\alpha \in \Lambda} P_{\alpha}=\left((0):{ }_{R} x\right) \cup\left((0):{ }_{R} y\right)$. Now $\left((0):{ }_{R} x\right) \cap(R \backslash Z(R))=\emptyset$. Hence by [19, Theorem 2.2, Page 378], we obtain that there exists a maximal $N$-prime $P$ of $(0)$ in $R$ such that $\left((0):_{R} x\right) \subseteq P$. Since $\left((0):_{R} y\right) \cap(R \backslash Z(R))=\emptyset$, it follows that there exists a maximal $N$-prime $Q$ of $(0)$ in $R$ such that $\left((0):_{R} y\right) \subseteq Q$. Now we obtain that $Z(R)=$ $\left((0):_{R} x\right) \cup\left((0):_{R} y\right)=P \cup Q$. If $P=Q$, then it follows that $\left((0):_{R} x\right) \cup\left((0):_{R} y\right)=P$ is an ideal of $R$. Hence it follows that either $\left((0):_{R} x\right) \subseteq\left((0):_{R} y\right)$ or $\left((0):_{R} y\right) \subseteq\left((0):_{R} x\right)$, and so we obtain that $P$ is the only maximal $N$-prime of $(0)$ in $R$ and either $P=\left((0):_{R} x\right)$ or $P=$ $\left((0)::_{R} y\right)$. Now by hypothesis $(\Gamma(R))^{c}$ is connected. Hence it follows from [18, Theorem 1.1 (a)] that $P$ is not a $B$-prime of $(0)$ in $R$ and so $P \neq Q$. Now we obtain from $Z(R)=\left((0):_{R} x\right) \cup$ $((0): R \quad y)=P \cup Q$ that $P$ and $Q$ are the only maximal $N$-primes of $(0)$ in $R$, and moreover, $P=\left((0):_{R} x\right)$ and $Q=\left((0):_{R} y\right)$.

The next lemma is [9, Lemma 3.6]. We make use of it in the proof of Lemma 2.4.

Lemma 2.3. Let $R$ be a ring. Let $P, Q$ be distinct B-prime ideals of $(0)$ in $R$ with $P=\left((0):_{R} x\right)$ and $Q=\left((0):_{R} y\right)$ for some $x, y \in R$. Then $x y=0$. 
Proof. For the sake of completeness, we give below an argument for the fact that $x y=0$. Since $P \neq Q$. either $P \not \subset Q$ or $Q \not \subset P$. Without loss of generality, we may assume that $P \not \subset Q$. Let $w$ $\in P \backslash Q$. Now $w x=0 \in Q$ and as $w \notin Q$, it follows that $x \in Q=((0): R \quad y)$. Hence $x y=0$.

We provide in the next lemma some sufficient conditions on $R$ in order that $(\Gamma(R))^{c}$ admits vertices $x$ such that $e(x)=3$ in $(\Gamma(R))^{c}$.

Lemma 2.4. Let $R$ be a ring and let $\left|Z(R)^{*}\right| \geq 2$. Suppose that $R$ has exactly two maximal $N$-primes of $(0)$ and let them be $P_{1}$ and $P_{2}$. If $(\Gamma(R))^{c}$ is connected and if $P_{1}=\left((0):_{R} u\right)$ and $P_{2}=\left((0):_{R} v\right)$ for some $u, v \in R$, then $d(u, v)=3$ in $(\Gamma(R))^{c}$ and so $e(u)=e(v)=3$ in $(\Gamma(R))^{c}$.

Proof. The proof of this lemma is contained in the proof of [18, Proposition 1.7], though it was not stated there in the above form. For the sake of completeness, we include a proof of it here.

We know from Lemma 2.3 that $u v=0$. Thus $u, v \in Z(R)^{*}$ and $u$ and $v$ are not adjacent in $(\Gamma(R))^{c}$. Since $P_{1}$ and $P_{2}$ are the only maximal $N$-primes of $(0)$ in $R$, it follows that $Z(R)=$ $P_{1} \cup P_{2}=\left((0):_{R} \quad u\right) \cup\left((0):_{R} v\right)$. Thus for any $w \in Z(R)^{*}$, either $w u=0$ or $w v=0$. This shows that $d(u, v) \geq 3$ in $(\Gamma(R))^{c}$. It is shown in the proof of [18, Proposition 1.7] that $d(x, y) \leq$ 3 in $(\Gamma(R))^{c}$ for any $x, y \in Z(R)^{*}$. Hence we obtain that $d(u, v)=3$ in $(\Gamma(R))^{c}$ and so it follows that $e(u)=e(v)=3$ in $(\Gamma(R))^{c}$.

We next state and prove the main theorem of this section.

Theorem 2.5. Let $R$ be a ring and let $\left|Z(R)^{*}\right| \geq 2$. If $(\Gamma(R))^{c}$ is connected, then the radius of $(\Gamma(R))^{c}$ is equal to 2 .

Proof. It is well known that $\Gamma(R)$ is connected [1, Theorem 2.3]. Hence it follows from Lemma 2.1 that for any $x \in Z(R)^{*}, e(x) \geq 2$ in $(\Gamma(R))^{c}$.

If $R$ has either exactly one maximal $N$-prime of $(0)$ or more than two maximal $N$ primes of (0), then it follows from Lemma 2.2. that for any $x \in Z(R)^{*}, e(x) \leq 2$ in $(\Gamma(R))^{c}$. Thus we obtain that if $R$ has either exactly one maximal $N$-prime of $(0)$ or more than two maximal $N$-primes of $(0)$, then for any $x \in Z(R)^{*}, e(x)=2$ in $(\Gamma(R))^{c}$. Hence we obtain in the cases mentioned above that $\operatorname{diam}\left((\Gamma(R))^{c}\right)=r\left((\Gamma(R))^{c}\right)=2$.

Assume that $R$ has exactly two maximal $N$-primes of (0) and let them be $P_{1}$ and $P_{2}$. In such a case, it was shown in the proof of [18, Proposition 1.7(i)] that there exist $a \in P_{1} \backslash P_{2}$ and $b \in P_{2} \backslash P_{1}$ such that $a b \neq 0$. It follows that $P_{1} \neq\left((0):_{R} a\right), P_{1} \neq\left((0):_{R} b\right)$, and $P_{2} \neq\left((0):_{R} a\right), P_{2} \neq\left((0):_{R} b\right)$. Now it follows from Lemma 2.2 that $d(a, y) \leq 2$ and $d(b, y) \leq 2$ for any $y \in Z(R)^{*}$ in $(\Gamma(R))^{c}$. Hence we obtain that $e(a) \leq 2$ and $e(b) \leq 2$ in $(\Gamma(R))^{c}$. Since $e(c) \geq 2$ in $(\Gamma(R))^{c}$ for any $c \in Z(R)^{*}$, it follows that $e(a)=e(b)=2$ in $(\Gamma(R))^{c}$. Thus in this case also, we arrive at the conclusion that the radius of $(\Gamma(R))^{c}$ is equal to 2 .

The following remark determines the center of $(\Gamma(R))^{c}$.

Remark 2.6. Let $R$ be a ring and let $\left|Z(R)^{*}\right| \geq 2$. Assume that $(\Gamma(R))^{c}$ is connected. In this remark, we determine the center of $(\Gamma(R))^{c}$.

If $R$ has either exactly one maximal $N$-prime of (0) or more than two maximal $N$-primes of (0), then it was shown in the proof of Theorem 2.5 that $\operatorname{diam}\left((\Gamma(R))^{c}\right)=$ $r\left((\Gamma(R))^{c}\right)=2$. Hence the center of $(\Gamma(R))^{c}$ is the set of all vertices of $(\Gamma(R))^{c}$. Moreover, if $R$ has exactly two maximal $N$-primes of $(0)$ and if at least one of them is not a $B$-prime of 
(0) in $R$, then it follows from Lemma 2.2 that for any $x \in Z(R)^{*}, e(x) \leq 2$ in $(\Gamma(R))^{c}$. Thus we obtain, in view of Lemma 2.1 that $e(x)=2$ for any $x \in Z(R)^{*}$. Hence in this case also we obtain that $\operatorname{diam}\left((\Gamma(R))^{c}\right)=r\left((\Gamma(R))^{c}\right)=2$ and so each vertex of $(\Gamma(R))^{c}$ is in the center of $(\Gamma(R))^{c}$.

Suppose that $R$ has exactly two maximal $N$-primes of (0) and both are $B$-primes of $(0)$ in $R$. Let $\left\{P_{1}, P_{2}\right\}$ be the set of all maximal $N$-primes of $(0)$ in $R$. Then it follows from Lemmas 2.1, 2.2, and 2.4 that the center of $(\Gamma(R))^{C}=\left\{x \in Z(R)^{*} \mid P_{1} \neq\left((0):_{R} x\right)\right.$ and $\left.P_{2} \neq\left((0):_{R} x\right)\right\}$.

We next present some examples to illustrate the results proved in this section.

Example 2.7. Let $V$ be a rank 1 valuation domain which is not discrete. Let $M$ denote the unique maximal ideal of $V$. Let $x \in M, \quad x \neq 0$. Let $R=V / x V$. Observe that $M / x V$ is the only prime ideal of $R$ and $Z(R)=M / x V$. Let us denote $M / x V$ by $P$. We assert that $P$ is not a $B$-prime of (0) in $R$. Suppose that $P$ is a $B$-prime of (0) in $R$. Then it can be easily verified that $M=(x V: V y)$ for some $y \in V$. Since $M \neq V$, it follows that $y \notin x V$. As $V$ is a valuation domain, we obtain that $x \in y V$. Thus $x=y v$ for some $v \in V$. Hence we obtain that $M=(x V: V \quad y)=(y v V: V y)=v V$. This is impossible since $M$ is not finitely generated. Thus $P$ is not a $B$-prime of $(0)$ in $R$. Now it follows from [18, Theorem 1.1 (a)] that $(\Gamma(R))^{c}$ is connected. We obtain from Theorem 2.5 that $\operatorname{diam}\left((\Gamma(R))^{c}\right)=r\left((\Gamma(R))^{c}\right)=2$ and each vertex of $(\Gamma(R))^{c}$ is in the center of $(\Gamma(R))^{c}$.

Example 2.8. (i) Let $R$ be as in Example 2.7 and let $T=R \times Z$ (resp. $T_{1}=R \times R$ ) be the direct product of $R$ and the ring of integers (resp. $R$ and $R$ ). We know from Example 2.7 that $P$ is the unique maximal $N$-prime of $(0)$ in $R$ and $P$ is not a $B$-prime of (0) in $R$. Note that $P_{1}=P \times Z$ and $P_{2}=R \times(0)$ (resp. $Q_{1}=P \times R$ and $Q_{2}=R \times P$ ) are the only maximal $N$-primes of the zero-ideal of $T$ (resp. of the zero-ideal of $T_{1}$ ) and $P_{1} \cap P_{2}=P \times(0)$ is not the zero-ideal of $T$ (resp. $Q_{1} \cap Q_{2}=P \times P$ is not the zero-ideal of $T_{1}$ ). Hence it follows from [18, Theorem 1.1(b)] that $(\Gamma(T))^{c}\left(\right.$ resp. $\left.\left(\Gamma\left(T_{1}\right)\right)^{c}\right)$ is connected. Since $P_{1}$ is not a $B$-prime of $(0)$ in $T$ (resp. $Q_{1}$ and $Q_{2}$ are not $B$-primes of $(0)$ in $\left.T_{1}\right)$, it follows from Remark 2.6 that diam $\left((\Gamma(T))^{c}\right)=r\left((\Gamma(T))^{c}\right)=2$ (resp. diam $\left(\left(\Gamma\left(T_{1}\right)\right)^{c}\right)=r\left(\left(\Gamma\left(T_{1}\right)\right)^{c}\right)=2$ ) and each vertex of $(\Gamma(T))^{c}$ is in the center of $(\Gamma(T))^{c}$ (resp. each vertex of $\left(\Gamma\left(T_{1}\right)\right)^{c}$ is in the center of $\left.\left(\Gamma\left(T_{1}\right)\right)^{c}\right)$.

(ii) Let $R=Z / 12 Z$. Note that $P_{1}=2 Z / 12 Z$ and $P_{2}=3 Z / 12 Z$ are the only prime ideals of the finite ring $R=Z / 12 Z$. Thus $Z(R)=P_{1} \cup P_{2}$. Observe that $R$ has exactly two maximal $N$-primes of (0) and $P_{1} \cap P_{2}=6 Z / 12 Z$ is not the zero-ideal of $R$. Hence it follows from $\left[18\right.$, Theorem 1.1(b)] that $(\Gamma(R))^{c}$ is connected. Moreover, observe that $2 Z=(12 Z: Z \pm$ $6 t)$ and $\{ \pm 6 t \mid t \in Z$ is odd and positive $\}$ is the set of all integers with the property that $2 Z=$ $(12 Z: Z \pm 6 t)$. Furthermore, note that $\{ \pm 4 k \mid k \in Z$ is positive and $k \equiv 1$ or $2(\bmod 3)\}$ is the set of all integers with the property that $3 Z=(12 Z: Z \pm 4 k)$. Hence it follows that $P_{1}=\left((0+12 Z):_{R} 6+12 Z\right), P_{2}=\left((0+12 Z):_{R} 4+12 Z\right)$, and $P_{2}=\left((0+12 Z):_{R} 8+12 Z\right)$. Thus $P_{1}$ and $P_{2}$ are $B$-primes of $(0)$ in $R$. Now it follows from [18, Proposition 1.7(b)] that $\operatorname{diam}\left((\Gamma(R))^{c}\right)=3$, and we know from Theorem 2.5 that $r\left((\Gamma(R))^{c}\right)=2$. Moreover, we obtain from Remark 2.6 and from the above discussion that the set of centers of $(\Gamma(R))^{c}=\{2+$ $12 Z, 10+12 Z, 3+12 Z, 9+12 Z\}$.

Example 2.9. Let $n>1$ be such that $n$ admits at least three distinct prime divisors. Let $\left\{p_{1}, p_{2}, p_{3}, \ldots, p_{t}\right\}(t \geq 3)$ be the set of all distinct prime divisors of $n$. Let $R=Z / n Z$. Note that $\left\{p_{1} Z / n Z, p_{2} Z / n Z, \ldots, p_{t} Z / n Z\right\}$ is the set of all maximal $N$-primes of the zero-ideal of $R$. We know from [18, Theorem 1.1(c)] that $(\Gamma(R))^{c}$ is connected and moreover, it is known 
from Remark 2.6 that $\operatorname{diam}\left((\Gamma(R))^{c}\right)=r\left((\Gamma(R))^{c}\right)=2$ and each vertex of $(\Gamma(R))^{c}$ is in the center of $(\Gamma(R))^{c}$.

\section{The Girth of $(\Gamma(R))^{c}$}

Let $R$ be a commutative ring with identity and let $\left|Z(R)^{*}\right| \geq 2$. The aim of this section is to study about the girth of $(\Gamma(R))^{c}$. If $(\Gamma(R))^{c}$ is connected, then we prove in this section that $\operatorname{gr}\left((\Gamma(R))^{c}\right)=3$. Moreover, we also discuss about the girth of $(\Gamma(R))^{c}$ in the case when $(\Gamma(R))^{c}$ is not connected.

For the sake of convenience we split the results proved in this section into several lemmas. We begin with the following lemma. We make use of this lemma in the proof of Lemma 3.2.

Lemma 3.1. Let $R$ be a ring and let $\left|Z(R)^{*}\right| \geq 2$. Let $P$ be a maximal $N$-prime of $(0)$ in $R$. If $P$ is not a $B$-prime of $(0)$ in $R$, then for any $x, y \in Z(R)^{*}, P \not \subset\left((0):_{R} x\right) \cup R y$.

Proof. Suppose that for some $x, y \in Z(R)^{*}, P \subseteq\left((0):_{R} x\right) \cup R y$. Then either $P \subseteq$ $\left((0):_{R} x\right)$ or $P \subseteq R y$. Since $x \neq 0,\left((0):_{R} x\right) \cap(R \backslash Z(R))=\emptyset$. As $y \in Z(R)$, there exists $z \in R \backslash\{0\}$ such that $y z=0$. Hence we obtain that $R y \subseteq\left((0):_{R} z\right)$. Note that $\left((0):_{R} z\right) \cap(R \backslash Z(R))=\emptyset$. Now it follows from [19, Theorem 2.2, Page 378] that there exists a maximal $N$-prime $Q$ of (0) in $R$ such that $\left((0):_{R} x\right) \subseteq Q$ and there exists a maximal $N$-prime $W$ of $(0)$ in $R$ such that $\left((0)::_{R} z\right) \subseteq W$. If $P \subseteq\left((0):_{R} x\right)$, then we obtain that $P \subseteq\left((0):_{R} x\right) \subseteq Q$ and hence it follows that $P=Q=\left((0):_{R} x\right)$. This contradicts the assumption that $P$ is not a $B$-prime of $(0)$ in $R$. If $P \subseteq R y$, then $P \subseteq\left((0):_{R} z\right) \subseteq W$ and so $P=W=\left((0):_{R} z\right)$. This is also impossible since $P$ is not a $B$-prime of $(0)$ in $R$. This proves that if a maximal $N$-prime $P$ of $(0)$ in $R$ is not a $B$-prime of (0) in $R$, then for any $x, y \in Z(R)^{*}, P \not \subset\left((0):_{R} x\right) \cup R y$.

In the following lemma, we determine the girth of $(\Gamma(R))^{c}$ under the assumptions that $R$ has exactly one maximal $N$-prime of $(0)$ and $(\Gamma(R))^{c}$ is connected.

Lemma 3.2. Let $R$ be a ring and let $\left|Z(R)^{*}\right| \geq 2$. Suppose that $R$ has only one maximal $N$-prime of (0). If $(\Gamma(R))^{c}$ is connected, then $\operatorname{gr}\left((\Gamma(R))^{c}\right)=3$.

Proof. Let $P$ be the unique maximal $N$-prime of $(0)$ in $R$. Since $(\Gamma(R))^{c}$ is connected, we obtain from [18, Theorem 1.1(a)] that $P$ is not a $B$-prime of $(0)$ in $R$. Note that $Z(R)=P$. Let $x \in$ $P \backslash\{0\}$. By hypothesis, $P$ is not a $B$-prime of (0) in $R$ and so Lemma 3.1 implies that there exists $y \in P$ such that $y \notin R x$ and $y x \neq 0$. We assert that $x y \notin\{x, y\}$. If $x y=x$, then $x(1-y)=0$. As $y \in P, 1-y \notin P=Z(R)$. Hence $x(1-y)=0$ implies that $x=0$. This contradicts the fact that $x \neq 0$. Similarly, it follows that $x y \neq y$. If both $x^{2} y$ and $y^{2} x$ are nonzero, then we obtain that $x-x y-y-x$ is a cycle of length 3 in $(\Gamma(R))^{c}$. Suppose that either $x^{2} y=0$ or $y^{2} x=0$. Without loss of generality, we may assume that $x^{2} y=0$. As $P$ is not a $B$-prime of (0) in $R$, Lemma 3.1 implies that there exists $z \in P$ such that $z x y \neq 0$ and $z \notin R y$. From $z x y \neq 0$, it follows that $z x \neq 0$, and $z y \neq 0$ and moreover, as $x^{2} y=0$, it follows that $z \neq x$. Furthermore, by the choice of $z$, it follows that $z \neq y$. Now $x-z-y-x$ is a cycle of length 3 in $(\Gamma(R))^{c}$. This shows that if $R$ has only one maximal $N$-prime of $(0)$ and if $(\Gamma(R))^{c}$ is connected, then there exists a cycle of length 3 in $(\Gamma(R))^{c}$ and hence $\operatorname{gr}\left((\Gamma(R))^{c}\right)=3$. Though the following lemma is elementary, we include it for the sake of future
reference. Though the following lemma is elementary, we include it for the sake of future
reference. 
Lemma 3.3. Let $R$ be a commutative ring with identity. If there exist distinct elements $a, b, c \in$ $Z(R)^{*} \backslash P$ for some prime ideal $P$ of $R$, then $\operatorname{gr}\left((\Gamma(R))^{c}\right)=3$.

Proof. As $P$ is a prime ideal of $R$ and $a, b, c$ are elements of $R$ which are not in $P$, we obtain that $a b, b c, c a \in R \backslash P$ and so $a b, b c, c a \in R \backslash\{0\}$. Hence it follows that $a-b-c-a$ is a cycle of length 3 in $(\Gamma(R))^{c}$. This proves that $\operatorname{gr}\left((\Gamma(R))^{c}\right)=3$.

The next lemma discusses the girth of $(\Gamma(R))^{c}$ where $R$ is a ring with (0) of $R$ admitting exactly two maximal $N$-primes.

Lemma 3.4. Let $R$ be a commutative ring with identity. Suppose that $R$ has exactly two maximal $N$-primes of (0) and let them be $P_{1}$ and $P_{2}$. Then the following hold:

(i) If $P_{1} \cap P_{2}=(0)$, then $(\Gamma(R))^{c}$ contains a cycle if and only if either $\left|P_{1} \backslash P_{2}\right| \geq 3$ or $\left|P_{2} \backslash P_{1}\right| \geq 3$ if and only if $\operatorname{gr}\left((\Gamma(R))^{c}\right)=3$.

(ii) If $P_{1} \cap P_{2} \neq(0)$, then $\operatorname{gr}\left((\Gamma(R))^{c}\right)=3$. Thus, if $(\Gamma(R))^{c}$ is connected, then $\operatorname{gr}\left((\Gamma(R))^{c}\right)=3$.

Proof. By hypothesis, $P_{1}$ and $P_{2}$ are the only maximal $N$-primes of (0) in $R$. So, it follows that $Z(R)=P_{1} \cup P_{2}$.

(i) Assume that $(\Gamma(R))^{c}$ contains a cycle. Let $a_{1}-a_{2}-a_{3}-\cdots-a_{n}-a_{1}$ be a cycle of length $n$ in $(\Gamma(R))^{c}$. Note that $n \geq 3$ and $\left\{a_{i} \mid i=1,2,3, \ldots, n\right\} \subseteq Z(R)^{*}$ with $a_{1} a_{2}, a_{2} a_{3}, \ldots, a_{n-1} a_{n}, a_{n} a_{1} \in R \backslash\{0\}$. Since $P_{1} \cap P_{2}=(0)$, it follows that either $\left\{a_{1}, a_{2}, a_{3}, \ldots, a_{n}\right\} \subseteq P_{1} \backslash P_{2}$ or $\left\{a_{1}, a_{2}, a_{3}, \ldots, a_{n}\right\} \subseteq P_{2} \backslash P_{1}$. Now it is clear that either $\left|P_{1} \backslash P_{2}\right| \geq 3$ or $\left|P_{2} \backslash P_{1}\right| \geq 3$.

Conversely, suppose that either $\left|P_{1} \backslash P_{2}\right| \geq 3$ or $\left|P_{2} \backslash P_{1}\right| \geq 3$. Since $Z(R)=P_{1} \cup P_{2}$ and as $P_{1}$ and $P_{2}$ are prime ideals of $R$, it follows from Lemma 3.3 that $\operatorname{gr}\left((\Gamma(R))^{c}\right)=3$.

If $\operatorname{gr}\left((\Gamma(R))^{c}\right)=3$, then we obtain that $(\Gamma(R))^{c}$ contains a cycle of length 3 .

(ii) Suppose that $P_{1} \cap P_{2} \neq(0)$. We know from the proof of [18, Proposition 1.7(i)] that there exist $a \in P_{1} \backslash P_{2}$ and $b \in P_{2} \backslash P_{1}$ such that $a b \neq 0$. We consider two cases:Case(A): $P_{1} \cap P_{2} \not \subset\left((0):_{R} a\right) \cup\left((0):_{R} b\right)$. Then there exists $c \in P_{1} \cap P_{2}$ such that $a c \neq 0$ and $b c \neq 0$. Hence we obtain a cycle $a-b-c-a$ in $(\Gamma(R))^{c}$ and it is of length 3. Case(B): $P_{1} \cap P_{2} \subseteq\left((0):{ }_{R} a\right) \cup$ $\left((0):{ }_{R} b\right)$. Then either $P_{1} \cap P_{2} \subseteq\left((0):{ }_{R} a\right)$ or $P_{1} \cap P_{2} \subseteq\left((0):{ }_{R} b\right)$.

Without loss of generality, we may assume that $P_{1} \cap P_{2} \subseteq\left((0):{ }_{R} a\right)$. Let $x \in P_{1} \cap P_{2}$ be such that $x \neq 0$. Since $a+b \notin Z(R)$, we obtain that $(a+b) x \neq 0$. As $a x=0$, it follows that $b x=(a+b) x \neq 0$. Moreover, observe that $b+x \in P_{2} \backslash P_{1}, \quad b \neq b+x$, and $a(b+x)=a b \neq 0$. As $b(b+x) \in P_{2} \backslash P_{1}$, it follows that $b(b+x) \neq 0$. Note that $a-b-b+x-a$ is a cycle of length 3 in $(\Gamma(R))^{c}$.

Thus in both the cases, $(\Gamma(R))^{c}$ admits a cycle of length 3 . Hence we obtain that $\operatorname{gr}\left((\Gamma(R))^{c}\right)=3$.

We know from [18, Theorem 1.1(b)] that $(\Gamma(R))^{c}$ is connected if and only if $P_{1} \cap$ $P_{2} \neq(0)$. Thus if $R$ has exactly two maximal $N$-primes of $(0)$ and if $(\Gamma(R))^{c}$ is connected, then $\operatorname{gr}\left((\Gamma(R))^{c}\right)=3$.

We show in the next lemma that if $R$ has at least three maximal $N$-primes of (0), then $\operatorname{gr}\left((\Gamma(R))^{c}\right)=3$.

Lemma 3.5. Let $R$ be a ring. Suppose that $R$ admits more than two maximal $N$-primes of $(0)$. Then $\operatorname{gr}\left((\Gamma(R))^{c}\right)=3$. 
Proof. Since by the assumption that $R$ admits more than two maximal $N$-primes of $(0)$, we can find at least three maximal $N$-primes of (0) in $R$. Let $\{P, Q, W\}$ be a subset of the set of all maximal $N$-primes of (0) in $R$. It is clear that $P \not \subset Q \cup W, Q \not \subset P \cup W$, and $W \not \subset P \cup Q$. Hence there exist elements $x \in P \backslash(Q \cup W), y \in Q \backslash(P \cup W)$, and $z \in W \backslash(P \cup Q)$. Note that $x, y, z$ are distinct elements of $Z(R)^{*}$ with $x y, y z, z x \in R \backslash\{0\}$. Hence $x-y-z-x$ is a cycle of length 3 in $(\Gamma(R))^{c}$. This proves that $\operatorname{gr}\left((\Gamma(R))^{c}\right)=3$.

With the help of the above lemmas, we obtain the main theorem of this section.

Theorem 3.6. Let $R$ be a ring and let $\left|Z(R)^{*}\right| \geq 2$. If $(\Gamma(R))^{c}$ is connected, then $\operatorname{gr}\left((\Gamma(R))^{c}\right)=3$.

Proof. The proof of this theorem follows immediately from [18, Theorem 1.1] and Lemmas 3.2, 3.4(ii), and 3.5.

We next proceed to consider rings $R$ such that $(\Gamma(R))^{c}$ is not connected and discuss about the girth of $(\Gamma(R))^{c}$. In this direction, we first have the following proposition.

Proposition 3.7. Let $R$ be a ring and let $\left|Z(R)^{*}\right| \geq 2$. Suppose that $R$ has only one maximal $N$-prime of $(0)$ and let it be $P$. If $P^{2} \neq(0)$ and if $(\Gamma(R))^{c}$ is not connected, then $(\Gamma(R))^{c}$ contains a cycle if and only if $|P| \geq 5$ if and only if $\operatorname{gr}\left((\Gamma(R))^{c}\right)=3$.

Proof. Note that $Z(R)=P$. Assume that $P^{2} \neq(0)$ and $(\Gamma(R))^{c}$ is not connected. From the assumption that $P^{2} \neq(0)$, it follows that there exist $a, b \in P$ such that $a \neq b$ and $a b \neq 0$. Moreover, by [18, Theorem 1.1(a)], $P=\left((0):_{R} c\right)$ for some $c \in R$. It is clear that $c \in P \backslash\{0\}$. Furthermore, as $a b \neq 0$, it follows that $a \neq c$ and $b \neq c$.

Suppose that $(\Gamma(R))^{c}$ contains a cycle. Let $a_{1}-a_{2}-a_{3}-\cdots-a_{n}-a_{1}$ be a cycle of length $n$ in $(\Gamma(R))^{c}$. Note that $n \geq 3$ and $\left\{a_{1}, a_{2}, a_{3}, \ldots, a_{n}\right\} \subseteq P \backslash\{0\}$. As $P=\left((0):_{R} c\right)$ and since $a_{1} a_{2}, a_{2} a_{3}, \ldots, a_{n-1} a_{n}$, and $a_{n} a_{1} \in R \backslash\{0\}$, it follows that $c \notin\left\{a_{1}, a_{2}, a_{3}, \ldots, a_{n}\right\}$. Thus $\left\{0, c, a_{1}, a_{2}, a_{3}, \ldots, a_{n}\right\} \subseteq P$ and hence it follows that $|P| \geq 5$.

We next show that $\operatorname{gr}\left((\Gamma(R))^{c}\right)=3$ if $|P| \geq 5$. Suppose that $|P| \geq 5$. Observe that there exists $d \in P \backslash\{0, a, b, c\}$ where $a, b, c$ are as in the first paragraph of this proof. We claim that there exists a cycle of length 3 in $(\Gamma(R))^{c}$. If $a d \neq 0$ and $b d \neq 0$, then $a-d-b-a$ is a cycle of length 3 in $(\Gamma(R))^{c}$. Suppose that either $a d=0$ or $b d=0$.

Observe that if $a^{2}=0$ and $b^{2}=0$, then from $(a+b) a=a b=(a+b) b=a b$, and from the fact that $a+b \in P \backslash\{0, a, b\}$, it follows that $a-a+b-b-a$ is a cycle of length 3 in $(\Gamma(R))^{c}$. Now let us suppose without loss of generality that $a^{2} \neq 0$. As $c^{2}=0$ and $a^{2} \neq 0$, it follows that $a+c \neq 0$. If $a+c \neq b$, then $a-a+c-b-a$ is a cycle of length 3 in $(\Gamma(R))^{c}$. Suppose that $a+c=b$. Then, as $c d=0$, it follows from the assumption that either $a d=0$ or $b d=0$ that $a d=b d=0$. As $c \neq d$ and $a+c=b$, it follows that $a+d \neq b$ and it is clear that $a(a+d)=a^{2} \neq$ 0 and $(a+d) b=a b \neq 0$. Hence $a-a+d-b-a$ is a cycle of length 3 in $(\Gamma(R))^{c}$.

Thus if $|P| \geq 5$, then it is shown that there exists a cycle of length 3 in $(\Gamma(R))^{c}$. Hence $\operatorname{gr}\left((\Gamma(R))^{c}\right)=3$.

If $\operatorname{gr}\left((\Gamma(R))^{c}\right)=3$, then $(\Gamma(R))^{c}$ contains a cycle of length 3 .

The following remark characterizes rings $R$ satisfying the following conditions: (i) $R$ has exactly one maximal $N$-prime of $(0),\left(\right.$ ii) $(\Gamma(R))^{c}$ contains at least one edge, (iii) $(\Gamma(R))^{c}$ is not connected, and (iv) $(\Gamma(R))^{c}$ does not contain any cycle.

Remark 3.8. Let $R$ be a ring and let $\left|Z(R)^{*}\right| \geq 2$. Suppose that $P$ is the only maximal $N$ prime of $(0)$ in $R$. Assume that $P^{2} \neq(0)$. Suppose that $(\Gamma(R))^{c}$ is not connected. Let $a, b \in P$ 
be such that $a \neq b$ and $a b \neq 0$. Let $c \in P \backslash\{0\}$ be such that $P=\left((0):_{R} c\right)$. Assume that $\operatorname{gr}\left((\Gamma(R))^{c}\right) \neq 3$. Observe that by Proposition 3.7, $\operatorname{gr}\left((\Gamma(R))^{c}\right) \neq 3$ if and only if $(\Gamma(R))^{c}$ does not contain any cycle. Now it follows from Proposition 3.7 that $|P| \leq 4$ and as $\{0, a, b, c\} \subseteq P$, it follows that $P=\{0, a, b, c\}$. Now $a b \in P \backslash\{0\}=\{a, b, c\}$. We assert that $a b=c$. If $a b=a$, then $a(1-b)=0$. This is impossible since $a \neq 0$ and $1-b \notin P=Z(R)$. Similarly, it follows that $a b \neq b$. Hence $a b=c$. Now $Z(R)=P=\{0, a, b, c=a b\}$ is finite and hence we obtain from [20, Theorem 1] that $R$ is a finite ring. We verify in this remark that $|R|=8$. Moreover, we observe with the help of [3, Theorem 3.2] that $R$ is isomorphic to exactly one of the rings from the set $\left\{Z_{8}, Z_{4}[x] /\left(2 x Z_{4}[x]+\left(x^{2}-2\right) Z_{4}[x]\right), Z_{2}[x] / x^{3} Z_{2}[x]\right\}$ where $Z_{4}[x]$ (resp. $\left.Z_{2}[x]\right)$ is the polynomial ring in one variable over $Z_{4}$ (resp. over $Z_{2}$ ).

Since $R$ is a finite ring, any prime ideal of $R$ is a maximal ideal of $R$, and moreover, if $Q$ is any prime ideal of $R$, then $Q \subseteq Z(R)$. Since $Z(R)=P$, it follows that $P$ is the only prime ideal of $R$. Now $R$ is a local ring with unique maximal ideal $P$. Hence we obtain that $R \backslash P$ is the set of all units in $R$. Let $u$ be a unit in $R$. Then $u a b \in P \backslash\{0\}=\{a, b, c=a b\}$. We claim that $u a b=a b$. If $u a b=a$, then $a(1-u b)=0$ and this is impossible. Similarly, we obtain that $u a b \neq b$. Hence $u a b=a b$. This implies that $(u-1) a b=0$. Since $a b \neq 0$, it follows that $u-1 \in Z(R)=P=\{0, a, b, c=a b\}$. Hence we obtain that $u \in\{1,1+a, 1+b, 1+c=1+a b\}$. Thus it is shown that $R \backslash P=\{1,1+a, 1+b, 1+c\}$. Hence $R=\{0, a, b, c=a b, 1,1+a, 1+b, 1+c\}$ is a ring containing exactly 8 elements. Note that $\omega(\Gamma(R))=2$. Now $[3$, Theorem 3.2] implies that $R$ is isomorphic to exactly one of the rings from the set $\left\{Z_{8}, Z_{4}[x] /\left(2 x Z_{4}[x]+\left(x^{2}-\right.\right.\right.$ 2) $\left.\left.Z_{4}[x]\right), \quad Z_{2}[x] / x^{3} Z_{2}[x]\right\}$. Observe that if $R$ is a ring such that $R \in\left\{Z_{8}, Z_{4}[x] /\left(2 x Z_{4}[x]+\right.\right.$ $\left.\left.\left(x^{2}-2\right) Z_{4}[x]\right), \quad Z_{2}[x] / x^{3} Z_{2}[x]\right\}$, then $R$ satisfies the following conditions: $R$ has exactly one maximal $N$-prime of $(0)$, say $P$ such that $P^{2} \neq(0),(\Gamma(R))^{c}$ is not connected, and $(\Gamma(R))^{c}$ does not contain any cycle.

Let $R$ be a ring such that $R$ has only one maximal $N$-prime of (0), say $P$, and $R$ satisfies the further conditions that $P^{2} \neq(0)$ and $(\Gamma(R))^{c}$ is not connected. The above discussion implies that $(\Gamma(R))^{c}$ does not contain any cycle if and only if $R$ is isomorphic to exactly one of the rings from the set $\left\{Z_{8}, Z_{4}[x] /\left(2 x Z_{4}[x]+\left(x^{2}-2\right) Z_{4}[x]\right), Z_{2}[x] / x^{3} Z_{2}[x]\right\}$.

We determine in the following remark rings $R$ satisfying the following conditions: (i) $R$ admits exactly two maximal $N$-primes of $(0)$, (ii) $(\Gamma(R))^{c}$ contains at least one edge, (iii) $(\Gamma(R))^{c}$ is not connected, and (iv) $(\Gamma(R))^{c}$ does not contain any cycle.

Remark 3.9. Let $R$ be a ring admitting exactly two maximal $N$-primes of (0). Let them be $P_{1}$ and $P_{2}$. Suppose that $P_{1} \cap P_{2}=(0)$ (that is, equivalently $(\Gamma(R))^{c}$ is not connected). In such a case, it is shown in Lemma 3.4(i) that $(\Gamma(R))^{c}$ contains a cycle if and only if either $\left|P_{1} \backslash P_{2}\right| \geq 3$, or $\left|P_{2} \backslash P_{1}\right| \geq 3$ if and only if $\operatorname{gr}\left((\Gamma(R))^{c}\right)=3$. Suppose that $\left|P_{1} \backslash P_{2}\right|<3,\left|P_{2} \backslash P_{1}\right|<3$ and that $(\Gamma(R))^{c}$ contains at least one edge. We verify in this remark that either $|R|=9$ or $|R|=6$. Moreover, we verify that $R$ is isomorphic to $K_{1} \times K_{2}$ where $K_{1}$ and $K_{2}$ are fields with either $\left|K_{1}\right|=\left|K_{2}\right|=3$ or one of them contains exactly 3 elements and the other contains exactly 2 elements.

We are assuming that $(\Gamma(R))^{c}$ contains at least one edge. Since $Z(R)=P_{1} \cup P_{2}$ and $P_{1} \cap P_{2}=(0)$, it follows that at least one between $P_{1}$ and $P_{2}$ contains exactly 3 elements. This implies that either $\left|P_{1}\right|=\left|P_{2}\right|=3$ or exactly one between $P_{1}$ and $P_{2}$ contains exactly 3 elements and the other contains exactly 2 elements. Thus either $|Z(R)|=5$ or $|Z(R)|=4$. As $Z(R)$ is a finite set, it follows from [20, Theorem 1$]$ that $R$ is a finite ring. Since any prime ideal of a finite ring is a maximal ideal, it follows that $P_{1}$ and $P_{2}$ are maximal ideals of $R$. As $P_{1} \cap P_{2}=(0)$, it follows from the Chinese Remainder Theorem [21, Proposition 1.10] that $R \approx\left(R / P_{1}\right) \times\left(R / P_{2}\right)$ 
as rings. Thus we obtain from the above discussion that $R$ is isomorphic to the direct product of two fields $K_{1}$ and $K_{2}$ with either $\left|K_{1}\right|=\left|K_{2}\right|=3$ or one between $K_{1}$ and $K_{2}$ contains exactly 3 elements and the other contains exactly 2 elements. Hence we obtain that either $|R|=9$ or $|R|=6$ and $R$ is isomorphic to $K_{1} \times K_{2}$ where $K_{1}$ and $K_{2}$ are fields with either $\left|K_{1}\right|=\left|K_{2}\right|=3$ or one of them contains exactly 3 elements and the other contains exactly 2 elements. Conversely, if $R$ is isomorphic to $K_{1} \times K_{2}$ where $K_{1}$ and $K_{2}$ are fields with either $\left|K_{1}\right|=\left|K_{2}\right|=3$ or one of them contains exactly 3 elements and the other contains exactly 2 elements, then it is clear that $R$ has the following properties: $R$ admits exactly two maximal $N$-primes of $(0),(\Gamma(R))^{c}$ contains at least one edge, $(\Gamma(R))^{c}$ is not connected and it does not contain any cycle.

We next have the following corollary, the proof of which is immediate from the results proved in this section.

Corollary 3.10. (i) Let $R$ be an infinite ring. If there exist $x, y \in Z(R)^{*}$ such that $x y \neq 0$, then $\operatorname{gr}\left((\Gamma(R))^{c}\right)=3$.

(ii) Let $R$ be any ring admitting elements $a, b \in Z(R)^{*}$ such that $a b \neq 0$. Then $\operatorname{gr}\left((\Gamma(R[x]))^{c}\right)$ $=\operatorname{gr}\left((\Gamma(R[[x]]))^{c}\right)=3$ where $R[x]$ (resp. $\left.R[[x]]\right)$ is the polynomial (resp.the power series) ring in one variable over $R$.

\section{Cliques in $(\Gamma(R))^{c}$}

Let $R$ be a commutative ring with identity which is not an integral domain. In this section, we prove that if a ring $R$ admits more than one maximal $N$-prime of $(0)$, then the clique number of $(\Gamma(R))^{c}$ is finite if and only if $(\Gamma(R))^{c}$ does not contain any infinite clique if and only if $R$ is finite. Moreover, if a ring $R$ is such that $R$ has only one maximal $N$-prime of $(0)$, we obtain some necessary conditions in order that the clique number of $(\Gamma(R))^{c}$ is finite. Furthermore, if $R$ is either a Noetherian ring or a chained ring and if $(\Gamma(R))^{c}$ admits at least one edge (that is, there exist $x, y \in Z(R)^{*}$ with $x \neq y$ such that $\left.x y \neq 0\right)$, then it is shown that the clique number of $(\Gamma(R))^{c}$ is finite if and only if $(\Gamma(R))^{c}$ does not contain any infinite clique if and only if $R$ is finite.

We first prove some elementary lemmas which are of interest in their own right and which are useful in proving the main results of this section. We begin with the following lemma.

Lemma 4.1. Let $R$ be a commutative ring with identity which is not an integral domain. Let $P$ be any prime ideal of $R$. Then the following hold.

(i) If $(\Gamma(R))^{c}$ does not contain any infinite clique, then $Z(R) \backslash P$ is finite.

(ii) If $\omega\left((\Gamma(R))^{c}\right)$ is finite, then $Z(R) \backslash P$ is finite and indeed, $|Z(R) \backslash P| \leq \omega\left((\Gamma(R))^{c}\right)$.

Proof. (i) Suppose that $Z(R) \backslash P$ is infinite. Then we can choose an infinite sequence of distinct elements $x_{i} \in Z(R) \backslash P$. Since $P$ is a prime ideal of $R$ and as $x_{i} \notin P$ for $i=1,2,3, \ldots$, it follows that $x_{i} x_{j} \neq 0$ for all $i, j \in\{1,2,3, \ldots\}$. Observe that the subgraph of $(\Gamma(R))^{c}$ induced on $\left\{x_{i} \mid i=1,2,3, \ldots\right\}$ is an infinite clique. This contradicts the assumption that $(\Gamma(R))^{c}$ does not contain any infinite clique. Hence we obtain that $Z(R) \backslash P$ is finite.

(ii) Let $\omega\left((\Gamma(R))^{c}\right)=n$. We assert that $|Z(R) \backslash P| \leq n$. Suppose that $\{Z(R) \backslash P \mid \geq$ $n+1$. Let $\left\{x_{1}, \ldots, x_{n+1}\right\} \subseteq Z(R) \backslash P$. Then it is clear that the subgraph of $(\Gamma(R))^{c}$ induced on 
$\left\{x_{1}, \ldots, x_{n+1}\right\}$ is a clique. This is impossible since $\omega\left((\Gamma(R))^{c}\right)=n$. This shows that $|Z(R) \backslash P| \leq$ $n=\omega\left((\Gamma(R))^{c}\right)$.

Using Lemma 4.1 and prime avoidance [21, Proposition 1.11(i)], we obtain the following result.

Lemma 4.2. Let $R$ be a commutative ring with identity which is not an integral domain. Let $P$ be any prime ideal of $R$. Let $A=\{Q \mid Q$ is a prime ideal of $R$ such that $Q \subseteq Z(R)$ but $Q \not \subset P\}$. Then the following hold.

(i) If $(\Gamma(R))^{c}$ does not contain any infinite clique, then $A$ can admit only a finite number of elements which are pairwise incomparable under inclusion.

(ii) If $\omega\left((\Gamma(R))^{c}\right)$ is finite, then $A$ can admit at most $\omega\left((\Gamma(R))^{c}\right)$ elements which are pairwise incomparable under inclusion.

Proof. (i) Suppose that $(\Gamma(R))^{C}$ does not contain any infinite clique. We want to verify that $A$ can admit only a finite number of elements which are pairwise incomparable under inclusion. Suppose that there exist infinitely many elements in $A$ which are pairwise incomparable under inclusion. Hence there exist $Q_{i} \in A$ for each $i=1,2,3, \ldots$ with $Q_{i}$ and $Q_{j}$ not being comparable under inclusion for all $i, j \in\{1,2,3, \ldots\}$ with $i \neq j$. Now $Q_{i} \not \subset P$ for $i=1,2,3, \ldots$. Hence it is possible to choose $x_{1} \in Q_{1} \backslash P$. Let $i \geq 2$. Now it follows from prime avoidance [21, Proposition 1.11(i)] that there exists $x_{i} \in Q_{i} \backslash\left(P \cup Q_{1} \cup \cdots \cup Q_{i-1}\right)$. Observe that $\left\{x_{i} \mid i=1,2,3, \ldots\right\} \subseteq Z(R) \backslash P$. Hence we obtain that $Z(R) \backslash P$ is infinite. This contradicts Lemma 4.1(i). This proves that if $(\Gamma(R))^{c}$ does not contain any infinite clique, then $A$ can admit only a finite number of elements which are pairwise incomparable under inclusion.

(ii) Let $\omega\left((\Gamma(R))^{c}\right)=n$. Suppose that $A$ admits more than $n$ elements which are pairwise incomparable under inclusion. Let $\left\{Q_{1}, \ldots, Q_{n+1}\right\} \subseteq A$ be such that $Q_{i}$ and $Q_{j}$ are not comparable for all $i, j \in\{1,2, \ldots, n+1\}$ with $i \neq j$. Let $x_{1} \in Q_{1} \backslash P$. Let $i \in\{2, \ldots, n+1\}$. As in (i), we can choose $x_{i} \in Q_{i} \backslash\left(P \cup Q_{1} \cup \cdots \cup Q_{i-1}\right)$. Observe that $\left\{x_{i} \mid i=1,2, \ldots, n+1\right\} \subseteq$ $Z(R) \backslash P$. This implies that $|Z(R) \backslash P| \geq n+1>\omega\left((\Gamma(R))^{c}\right)$. This contradicts Lemma 4.1 (ii). Hence we obtain that $A$ can admit at most $n=\omega\left((\Gamma(R))^{c}\right)$ elements which are pairwise incomparable under inclusion.

We next study in the following corollary to Lemma 4.2, the effect of the nature of the cliques of $(\Gamma(R))^{c}$ on the set of maximal $N$-primes of $(0)$, and the set of minimal prime ideals of $R$.

Corollary 4.3. Let $R$ be a commutative ring with identity which is not an integral domain. Then the following hold.

(i) If $(\Gamma(R))^{c}$ does not contain any infinite clique then (a) the set of maximal $N$-primes of $(0)$ in $R$ is finite (b) the set of minimal prime ideals of $R$ is finite.

(ii) If $\omega\left((\Gamma(R))^{c}\right)$ is finite, then $(a) R$ can admit at most $\omega\left((\Gamma(R))^{c}\right)+1$ maximal $N$ primes of (0) and if $R$ admits exactly $k$ maximal $N$-primes of $(0)$ with $k \geq 3$, then $k \leq \omega\left((\Gamma(R))^{c}\right)(b) R$ can admit at most $\omega\left((\Gamma(R))^{c}\right)+1$ minimal prime ideals, and if $k$ is the number of minimal prime ideals of $R$ with $k \geq 3$, then $k \leq \omega\left((\Gamma(R))^{c}\right)$.

Proof. (i) (a) Let $P$ be a maximal $N$-prime of (0) in $R$. Let $A=\{Q \mid Q$ is a maximal $N$ prime of (0) in $R$ and $Q \neq P\}$. Since any maximal $N$-prime of (0) in $R$ is a subset of $Z(R)$ and as distinct maximal $N$-primes of $(0)$ in $R$ are not comparable under inclusion, it follows 
from Lemma 4.2(i) that $A$ is finite. Observe that the set of all maximal $N$-primes of $(0)$ in $R=A \cup\{P\}$. It is now clear that $R$ can admit only a finite number of maximal $N$-primes of $(0)$.

(i) (b) If $P$ is any minimal prime ideal of $R$, then $P \subseteq Z(R)$ [22, Theorem 84]. Since distinct minimal prime ideals of $R$ are not comparable under inclusion, it follows using the same arguments as in the proof of (i) (a) that $R$ can admit only a finite number of minimal prime ideals.

(ii)(a) Let $P, A$ be as in the proof of (i)(a). Let $\omega\left((\Gamma(R))^{c}\right)=n$. Now using the same arguments as in the proof of (i) (a), it follows from Lemma 4.2(ii) that $|A| \leq n$. Since the set of all maximal $N$-primes of (0) in $R=A \cup\{P\}$, we obtain that $R$ can admit at most $n+1$ maximal $N$-primes of (0).

Suppose that $R$ admits exactly $k$ maximal $N$-primes of (0) with $k \geq 3$. Let $\left\{P_{1}, P_{2}, P_{3}, \ldots, P_{k}\right\}$ be the set of all maximal $N$-primes of $(0)$ in $R$. Note that $Z(R)=$ $\cup_{i=1}^{k} P_{i}$. Since distinct maximal $N$-primes of $(0)$ in $R$ are not comparable under the inclusion relation, it follows from [21, Proposition 1.11(i)] that for each $i \in\{1,2,3, \ldots, k\}, \exists x_{i} \in$ $P_{i} \backslash\left(\bigcup_{j \in\{1, \ldots, k\} \backslash\{i\}} P_{j}\right)$. Then it is clear that for any distinct $i, j \in\{1,2,3, \ldots, k\}, x_{i}$ and $x_{j}$ are distinct nonzero zero-divisors of $R$, and as $k \geq 3$, it follows that there exists at least one $t \in\{1,2,3, \ldots, k\}$ such that both $x_{i}$ and $x_{j}$ are not in $P_{t}$ and hence $x_{i} x_{j} \neq 0$. Thus we obtain that the subgraph of $(\Gamma(R))^{c}$ induced on $\left\{x_{1}, x_{2}, x_{3}, \ldots, x_{k}\right\}$ is a clique and so $k \leq \omega\left((\Gamma(R))^{c}\right)$.

(ii)(b) This can be proved using similar arguments as in the proof of (ii)(a) and using Lemma 4.2(ii).

The following proposition is one among the main results in this section. We show in this proposition that if a ring $R$ admits at least two maximal $N$-primes of $(0)$, then $\omega\left((\Gamma(R))^{c}\right)$ is finite if and only if $R$ is finite.

Proposition 4.4. Let $R$ be a commutative ring with identity. Suppose that $R$ has at least two maximal $N$-primes of $(0)$. Then the following conditions are equivalent:

(i) $\omega\left((\Gamma(R))^{c}\right)$ is finite.

(ii) $R$ is finite.

(iii) $(\Gamma(R))^{c}$ does not contain any infinite clique.

Proof. (i) $\Rightarrow$ (ii) Let $\omega\left((\Gamma(R))^{c}\right)=n$. Now it follows from Corollary 4.3(ii)(a), that $R$ can admit at most $n+1$ maximal $N$-primes of (0). Let $m$ be the number of maximal $N$-primes of (0) in $R$ and let $\left\{P_{1}, \ldots, P_{m}\right\}$ be the set of all maximal $N$-primes of $(0)$ in $R$. Note that $m \leq n+1$ and by the hypothesis, $m \geq 2$. Observe that

$$
\begin{aligned}
Z(R) & =P_{1} \cup P_{2} \cup \cdots \cup P_{m} \\
& =\left(P_{1} \cap P_{2} \cap \cdots \cap P_{m}\right) \cup\left(Z(R) \backslash P_{1}\right) \cup\left(Z(R) \backslash P_{2}\right) \cup \cdots \cup\left(Z(R) \backslash P_{m}\right) .
\end{aligned}
$$

We know from Lemma 4.1(ii) that

$$
\left|Z(R) \backslash P_{i}\right| \leq n \quad \text { for } i=1,2, \ldots, m .
$$


We now verify that $\left|P_{1} \cap P_{2} \cap \cdots \cap P_{m}\right| \leq n$. Let $x \in P_{1} \backslash P_{2}$. Note that $\{x+y \mid y \in$ $\left.P_{1} \cap P_{2} \cap \cdots \cap P_{m}\right\} \subseteq Z(R) \backslash P_{2}$. Hence it follows that

$$
\left|P_{1} \cap P_{2} \cap \cdots \cap P_{m}\right| \leq n .
$$

Now it follows from (4.1), (4.2), and (4.3) that $Z(R)$ is finite. Hence it follows from [20, Theorem 1] that $R$ is finite.

(ii) $\Rightarrow$ (iii) This is obvious.

(iii) $\Rightarrow$ (i) We first show that $R$ is finite. It follows from Corollary 4.3(i)(a) that $R$ can admit only a finite number of maximal $N$-primes of $(0)$. Now one can proceed as in the proof of (i) $\Rightarrow$ (ii), and use Lemma 4.1(i) to obtain that $R$ is finite. It is now clear that $\omega\left((\Gamma(R))^{c}\right)$ is finite.

Remark 4.5. Let $R$ be an infinite ring and let $R$ admit at least two maximal $N$-primes of (0). It follows from Proposition 4.4 that $\omega\left((\Gamma(R))^{c}\right)=\infty$. Motivated by the interesting theorems proved on cliques in $\Gamma(R)$ in [3], and in particular, [3, Theorems 3.7 and 3.8], we attempt to determine $\omega\left((\Gamma(R))^{c}\right)$ for a finite commutative ring $R$ with identity which admits at least two maximal $N$-primes of $(0)$. We are able to describe $\omega\left((\Gamma(R))^{c}\right)$ in the case when $R$ is a finite reduced ring which is not an integral domain.

Let $R$ be a finite commutative reduced ring with identity which is not an integral domain. Since $R$ is a finite ring, any prime ideal of $R$ is maximal. Let $\left\{P_{1}, \ldots, P_{n}\right\}$ be the set of all prime ideals of $R$. Since $R$ is reduced, $(0)=\operatorname{nil}(R)=P_{1} \cap \cdots \cap P_{n}$. As $R$ is not an integral domain, it follows that $n \geq 2$. Moreover, by the Chinese Remainder Theorem [21, Proposition 1.10], it follows that $R$ is isomorphic to $R / P_{1} \times \cdots \times R / P_{n}$. Thus $R$ is isomorphic to a finite direct product of finite fields. Let $n \geq 2$ and let $K_{1} \ldots, K_{n}$ be finite fields. Let $R=K_{1} \times \cdots \times K_{n}$. We now proceed to describe $\omega\left((\Gamma(R))^{c}\right)$. We make use of some of the techniques from [4].

We first recall the following facts from [4].

Fact 4.6. Let $R$ be a commutative ring with identity which is not an integral domain. For any $a, b \in Z(R)^{*}$, define $a \sim b$ if and only if $\left((0):_{R} a\right)=\left((0):_{R} b\right)$. Then $\sim$ is an equivalence relation on $Z(R)^{*}$.

Proof. This fact is easy to check. The relation $\sim$ can be defined on the whole of $R$. As our interest is on $Z(R)^{*}$, we consider this relation defined on $Z(R)^{*}$.

For an element $a \in Z(R)^{*}$, we denote by $[a]$, the equivalence class determined by containing " $a$ ".

Recall from [23] that a commutative ring $R$ with identity is said to be von Neumann regular if for each $a \in R$ there exists $b \in R$ such that $a=a^{2} b$.

The following fact is important, and we make use of it in the proof of Lemma 4.8.

Fact 4.7. Let $R$ be a von Neumann regular ring which is not an integral domain (equivalently, which is not a field). Let $\sim$ be the relation defined on $Z(R)^{*}$ as in Fact 4.6. Then for any $a \in Z(R)^{*}$, there exists a unique idempotent element $e \in Z(R)^{*}$ such that $[a]=[e]$. 
Proof. The Fact 4.7 can be proved easily with the help of the ideas contained in the proof of [4, Lemma 3.1] and [10, Lemma 2.11]. In fact, [4, Lemma 3.1] and [10, Lemma 2.11] assert that for any von Neumann regular ring $R$ and for $a, b \in Z(R)^{*},[a]=[b]$ if and only if $R a=R b$. Yet for the sake of completeness, we present a proof of Fact 4.7.

It is well known that any element of a von Neumann regular ring can be expressed as the product of a unit and an idempotent [24, Lemma 2.5]. Let $a \in Z(R)^{*}$. Now there exists a unit $u$ in $R$ and an idempotent element $e$ in $R$ such that $a=u e$. Then it is clear that $\left((0):_{R} a\right)=\left((0):_{R} e\right)$. Since $a \in Z(R)^{*}$, it follows that $e \in Z(R)^{*}$. This proves that $a \sim e$ and so $[a]=[e]$.

Note that if $e$ is any idempotent element in a ring $R$ ( $R$ not necessarily von Neumann regular), then $\left((0):_{R} e\right)=R(1-e)$. Moreover, it is easy to check that for idempotent elements $e_{1}, e_{2}$ in a ring $R, R e_{1}=R e_{2}$ if and only if $e_{1}=e_{2}$. If $e, f$ are idempotent elements in a ring $R$ such that $\left((0):_{R} e\right)=\left((0):_{R} f\right)$, then it follows that $R(1-e)=R(1-f)$. Hence $1-e=1-f$ and so $e=f$.

Now it follows from the above two preceding paragraphs that given any $a \in Z(R)^{*}$ where $R$ is a von Neumann regular ring, then there exists a unique idempotent $e \in Z(R)^{*}$ such that $[a]=[e]$.

Let $R$ be a von Neumann regular ring which is not an integral domain. In the following lemma, we exhibit some cliques of $(\Gamma(R))^{c}$.

Lemma 4.8. Let $R$ be a von Neumann regular ring which is not an integral domain. Let $\left\{e_{1}, \ldots, e_{m}\right\} \subseteq R \backslash\{0,1\}$ be a set of idempotents in $R$ such that $e_{i} e_{j} \neq 0$ for all $i, j \in\{1, \ldots, m\}$. Let $A=\left[e_{1}\right] \cup \cdots \cup\left[e_{m}\right]$. Then the subgraph of $(\Gamma(R))^{c}$ induced on $A$ is a clique.

Proof. Let $i \in\{1, \ldots, m\}$. Let $a, b \in\left[e_{i}\right]$ with $a \neq b$. Note that $[a]=[b]=\left[e_{i}\right]$. Now it follows from the proof of Fact 4.7 that there exist units $u, v$ in $R$ such that $a=u e_{i}$ and $b=v e_{i}$. Hence $a b=u v e_{i} \neq 0$. This proves that any two distinct elements of $\left[e_{i}\right]$ are adjacent in $(\Gamma(R))^{c}$ for each $i \in\{1, \ldots, m\}$.

Let $i, j \in\{1, \ldots, m\}$ with $i \neq j$. Let $x \in\left[e_{i}\right], y \in\left[e_{j}\right]$. Note that $x=u e_{i}$ and $y=v e_{j}$ for some units $u, v$ in $R$. Hence $x y=u v e_{i} e_{j} \neq 0$, since $e_{i} e_{j} \neq 0$ by the hypothesis.

Indeed, it is true that if $[a]$ and $[b]$ are equivalence classes determined by $\sim$ defined on $Z(R)^{*}$ where $R$ is a commutative ring with identity which is not an integral domain ( $R$ need not be von Neumann regular) and if $a b \neq 0$, then we assert that for any $x \in[a]$ and $y \in[b]$, $x y \neq 0$. Note that if $x y=0$, then $y \in\left((0):_{R} x\right)=\left((0):_{R} a\right)$. Hence $a y=0$. This implies that $a \in\left((0):_{R} y\right)=\left((0):_{R} b\right)$. Hence $a b=0$ and this is a contradiction. Thus we obtain that $x y \neq 0$.

This proves that the subgraph of $(\Gamma(R))^{c}$ induced on $A=\left[e_{1}\right] \cup \cdots \cup\left[e_{m}\right]$ is a clique.

We include the following simple lemma for the sake of completeness.

Lemma 4.9. Let $n \geq 2$. Let $K_{i}$ be a field for $i=1,2, \ldots, n$. Let $R=K_{1} \times \cdots \times K_{n}$ be their direct product. Then the number of idempotents in $Z(R)^{*}$ (that is, the number of nontrivial idempotents in $R)$ equals $2^{n}-2$ and the number of equivalence classes determined by the equivalence relation $\sim$ defined on $Z(R)^{*}$ equals $2^{n}-2$.

Proof. The only idempotent elements in a field are 1 and 0 . Using this observation, it follows that $R=K_{1} \times \cdots \times K_{n}$ has exactly $2^{n}$ idempotents. Among them except $(0, \ldots, 0)$ and $(1, \ldots, 1)$, 
the rest of them are in $Z(R)^{*}$. Thus we obtain that the number of idempotents in $Z(R)^{*}$ (that is, the number of nontrivial idempotents in $R$ ) equals $2^{n}-2$.

Let $\left\{e_{i} \mid i=1,2, \ldots, 2^{n}-2\right\}$ be the set of all idempotent elements in $Z(R)^{*}$. Since $R=K_{1} \times \cdots \times K_{n}$ is von Neumann regular, it follows from Fact 4.7 that the set of all equivalence classes determined by $\sim$ is $\left\{\left[e_{i}\right] \mid i=1,2, \ldots, 2^{n}-2\right\}$.

Let $n \geq 2$. Let $R=K_{1} \times \cdots \times K_{n}$ where $K_{i}$ is a field for $i=1,2, \ldots, n$. The following lemma describes the cliques of $(\Gamma(R))^{c}$.

Lemma 4.10. Let $n \geq 2$. Let $K_{i}$ be a field for $i=1,2, \ldots, n$. Let $R=K_{1} \times \cdots \times K_{n}$. Let $A \subseteq Z(R)^{*}$ be such that the subgraph of $(\Gamma(R))^{c}$ induced on $A$ is a clique. Let $E=\left\{e_{i} \mid i=1,2, \ldots, 2^{n}-2\right\}$ be the set of all idempotents in $Z(R)^{*}$. Then there exists a nonempty subset $B$ of $E$ such that $b_{1} b_{2} \neq 0$ for all $b_{1}, b_{2} \in B$ and $A \subseteq \bigcup_{b \in B}[b]$.

Proof. We know from the proof of Lemma 4.9 that $\left\{\left[e_{i}\right] \mid i=1,2, \ldots, 2^{n}-2\right\}$ is the set of all equivalence classes determined by $\sim$. Thus we obtain that $Z(R)^{*}=\bigcup_{e \in E}[e]$. Now $A$ being a subset of $Z(R)^{*}$, it follows that $A=\bigcup_{e \in E}(A \cap[e])$. Let $B \subseteq E$ be such that $A \cap[b]$ is non-empty for each $b \in B$. Since $A$ is non-empty, it follows that $B$ is non-empty. We now verify that for any $b_{1}, b_{2} \in B, b_{1} b_{2} \neq 0$. This is clear if $b_{1}=b_{2}$ since any element of $B$ is a nonzero idempotent in $R$. Suppose that $b_{1} \neq b_{2}$.

Let $a_{1} \in A \cap\left[b_{1}\right]$ and $a_{2} \in A \cap\left[b_{2}\right]$. Since $\left[b_{1}\right] \cap\left[b_{2}\right]=\emptyset$, it follows that $a_{1} \neq a_{2}$. As the subgraph of $(\Gamma(R))^{c}$ induced on $A$ is a clique, we obtain that $a_{1} a_{2} \neq 0$. Note that $R a_{i}=$ $R b_{i}$ for $i=1,2$. Hence we obtain that $R b_{1} b_{2}=R a_{1} a_{2} \neq(0)$ and so $b_{1} b_{2} \neq 0$. This proves that there exists a non-empty subset $B$ of $E$ such that $b_{1} b_{2} \neq 0$ for all $b_{1}, b_{1} \in B$ and moreover, $A=\bigcup_{b \in B}(A \cap[b]) \subseteq \bigcup_{b \in B}[b]$.

Let $R$ be as in Lemma 4.10 with the further assumption that $K_{i}$ is finite for $i=$ $1,2, \ldots, n$. We determine $\omega\left((\Gamma(R))^{c}\right)$ in the following proposition.

Proposition 4.11. Let $n \geq 2$ and let $K_{i}(i=1,2, \ldots, n)$ be finite fields. Let $R=K_{1} \times K_{2} \times \cdots \times K_{n}$. Let $E=\left\{e_{i} \mid i=1,2, \ldots, 2^{n}-2\right\}$ be the set of all idempotents in $Z(R)^{*}$. Then $\omega\left((\Gamma(R))^{c}\right)=$ $\max \left\{\left(\sum_{b \in B}|[b]|\right) \mid B\right.$ varies over all non-empty subsets of $E$ satisfying the property that $b_{1} b_{2} \neq 0$ for any $\left.b_{1}, b_{2} \in B\right\}$.

Proof. Let $X=\left\{B \mid B\right.$ is a non-empty subset of $E$ satisfying the property that for any $b_{1}, b_{2} \in$ $\left.B, b_{1} b_{2} \neq 0\right\}$. Let $B \in X$. Let $A=\bigcup_{b \in B}[b]$. Now it follows from Lemma 4.8 that the subgraph of $(\Gamma(R))^{c}$ induced on $A$ is a clique. Thus $\omega\left((\Gamma(R))^{c}\right) \geq|A|=\sum_{b \in B}|[b]|$. Hence we obtain that

$$
\omega\left((\Gamma(R))^{c}\right) \geq \max \left\{\left(\sum_{b \in B}|[b]|\right) \mid B \in X\right\} .
$$

Let $A \subseteq Z(R)^{*}$ be such that the subgraph of $(\Gamma(R))^{c}$ induced on $A$ is a clique. We know from Lemma 4.10 that there exists $B \in X$ such that $A \subseteq \bigcup_{b \in B}[b]$. Hence we obtain that $|A| \leq \sum_{b \in B}|[b]|$. This implies that

$$
\omega\left((\Gamma(R))^{c}\right) \leq \max \left\{\left(\sum_{b \in B}|[b]|\right) \mid B \in X\right\} .
$$

From (4.4) and (4.5), it follows that $\omega\left((\Gamma(R))^{c}\right)=\max \left\{\left(\sum_{b \in B}|[b]|\right) \mid B \in X\right\}$. 
We make use of the following useful remark in Example 4.13(i) and (ii).

Remark 4.12. Let $R$ be a von Neumann regular ring which is not a field. Let $\sim$ be the equivalence relation which was considered in Fact 4.7. Observe that for any idempotent element $e \in Z(R)^{*},[e]=\{u e \mid u$ is a unit in $R\}$.

Let $n \geq 2$. Let $K_{1}, K_{2}, \ldots, K_{n}$ be finite fields and let $R=K_{1} \times K_{2} \times \cdots \times K_{n}$. Let $e \in Z(R)^{*}$ be an idempotent. Let $C=\{i \in\{1,2, \ldots, n\} \mid$ the $i$ th component of $e$ is 1$\}$. Observe that $|[e]|=\prod_{i \in C}\left(\left|K_{i}\right|-1\right)$.

We next have the following example.

Example 4.13. (i) Let $K_{1}, K_{2}$ be finite fields. Let $R=K_{1} \times K_{2}$. Note that $\left\{e_{1}=(1,0), e_{2}=\right.$ $(0,1)\}$ is the set of all idempotents in $Z(R)^{*}$. Now it follows from Proposition 4.11 and Remark 4.12 that $\omega\left((\Gamma(R))^{c}\right)=\max \left\{\left|K_{1}\right|-1,\left|K_{2}\right|-1\right\}$. Note that this fact can also be verified directly.(ii) Let $K_{1}, K_{2}, K_{3}$ be finite fields. Let $R=K_{1} \times K_{2} \times K_{3}$. Note that $\left\{e_{1}=\right.$ $\left.(1,0,0), e_{2}=(0,1,0), e_{3}=(0,0,1), e_{4}=(1,1,0), e_{5}=(1,0,1), e_{6}=(0,1,1)\right\}$ is the set of all idempotents in $Z(R)^{*}$. Now it follows from Proposition 4.11 and Remark 4.12 that $\omega\left((\Gamma(R))^{c}\right)=\max \left\{\left(\left|K_{1}\right|-1\right)+\left(\left|K_{1}\right|-1\right)\left(\left|K_{2}\right|-1\right)+\left(\left|K_{1}\right|-1\right)\left(\left|K_{3}\right|-1\right),\left(\left|K_{2}\right|-1\right)+\left(\left|K_{1}\right|-\right.\right.$ $1)\left(\left|K_{2}\right|-1\right)+\left(\left|K_{2}\right|-1\right)\left(\left|K_{3}\right|-1\right),\left(\left|K_{3}\right|-1\right)+\left(\left|K_{1}\right|-1\right)\left(\left|K_{3}\right|-1\right)+\left(\left|K_{2}\right|-1\right)\left(\left|K_{3}\right|-1\right),\left(\left|K_{1}\right|-\right.$ 1) $\left.\left(\left|K_{2}\right|-1\right)+\left(\left|K_{2}\right|-1\right)\left(\left|K_{3}\right|-1\right)+\left(\left|K_{1}\right|-1\right)\left(\left|K_{3}\right|-1\right)\right\}$.

Let $R$ be a ring with $\left|Z(R)^{*}\right| \geq 2$. Suppose that $R$ has only one maximal $N$-prime of (0). We, in the following result, determine some necessary conditions on $R$ in order that $(\Gamma(R))^{c}$ does not contain any infinite clique.

Lemma 4.14. Let $R$ be a commutative ring with identity and let $\left|Z(R)^{*}\right| \geq 2$. Suppose that $R$ has exactly one maximal $N$-prime of $(0)$ and let it be $P$. If $(\Gamma(R))^{c}$ does not contain any infinite clique, then (i) $P=$ the nilradical of $R$ and if furthermore, $P^{2} \neq(0)$, then (ii) $R / P$ is finite, (iii) $P$ is a B-prime of ( 0$)$ in $R$, and $(i v) R$ satisfies descending chain condition (d.c.c.) on principal ideals.

Proof. (i) As any nilpotent element of $R$ is a zero-divisor of $R$ and since $Z(R)=P$, it follows that $\operatorname{nil}(R) \subseteq Z(R)=P$. Let $x \in P$. We assert that $x$ is nilpotent. Suppose that $x$ is not nilpotent. Then for any $i, j \in\{1,2,3, \ldots\}, x^{i} x^{j} \neq 0$, and moreover, for all distinct $i, j \in\{1,2,3, \ldots\}, x^{i} \neq x^{j}$. Hence $\left\{x^{k} \mid k=1,2,3, \ldots\right\} \subseteq Z(R)^{*}$ is such that the subgraph of $(\Gamma(R))^{c}$ induced on $\left\{x_{k} \mid\right.$ $k=1,2,3, \ldots\}$ is an infinite clique. This is in contradiction to the assumption that $(\Gamma(R))^{c}$ does not contain any infinite clique. Hence $x$ is nilpotent. This shows that $P \subseteq \operatorname{nil}(R)$ and so $P=\operatorname{nil}(R)$.

(ii) Suppose that $R / P$ is infinite. We first assert that $x^{2}=0$ for each $x \in P$. Suppose that there exists $x \in P$ such that $x^{2} \neq 0$. Since we are assuming that $R / P$ is infinite, it is possible to find an infinite sequence of elements $r_{k} \in R \backslash P$ for $k=1,2,3, \ldots$ such that $r_{i}-r_{j} \notin P$ and $r_{i}+r_{j} \notin P$ for all distinct $i, j \in\{1,2,3, \ldots\}$. Note that for all $i, j \in\{1,2,3, \ldots\}, r_{i} r_{j} \notin P=$ $Z(R)$, and as $x^{2} \neq 0$, it follows that $x^{2} r_{i} r_{j} \neq 0$. Moreover, as for all distinct $i, j \in\{1,2,3, \ldots\}$, $r_{i}-r_{j} \notin P$, it follows that $x r_{i} \neq x r_{j}$. Hence we obtain that the subgraph of $(\Gamma(R))^{c}$ induced on $\left\{x r_{k} \mid k=1,2,3, \ldots\right\}$ is an infinite clique. This contradicts the assumption that $(\Gamma(R))^{c}$ does not contain any infinite clique. Hence $x^{2}=0$ for each $x \in P$. As $P^{2} \neq(0)$, there exist $a, b \in P$ such that $a \neq b$ and $a b \neq 0$. Note that we have $a^{2}=b^{2}=0$. For any positive integer $k$, let $x_{k}=a+b r_{k}$. It is clear that for any positive integer $k, x_{k} \in P$ and $a x_{k}=a b r_{k} \neq 0$, since 
$a b \neq 0$ and $r_{k} \notin P=Z(R)$. Moreover, for all distinct $i, j \in\{1,2,3, \ldots\}, x_{i} x_{j}=a b\left(r_{i}+r_{j}\right) \neq 0$, since $a b \neq 0$ and $r_{i}+r_{j} \notin P=Z(R)$. Furthermore, as $b \neq 0$ and $r_{i}-r_{j} \notin P$, for all distinct $i, j \in\{1,2,3, \ldots\}$, it follows that $x_{i} \neq x_{j}$ for all distinct $i, j \in\{1,2,3, \ldots\}$. Hence we obtain that the subgraph of $(\Gamma(R))^{c}$ induced on $\left\{x_{k} \mid k=1,2,3, \ldots\right\}$ is an infinite clique. This contradicts the assumption that $(\Gamma(R))^{c}$ does not contain any infinite clique. Hence it follows that $R / P$ is finite.

(iii) We now verify that $P$ is a $B$-prime of (0) in $R$. We consider two cases. Case (A): $P$ is finitely generated.

By (i), we have $P=\operatorname{nil}(R)$ and hence we obtain that $P$ is a nilpotent ideal of $R$. Let $m \geq 2$ be least with the property that $P^{m}=(0)$. Now for any $x \in P^{m-1} \backslash\{0\}, P \subseteq\left((0):_{R} x\right) \subseteq$ $Z(R)=P$, and so $P=\left((0):_{R} x\right)$ is a $B$-prime of $(0)$ in $R$. Case (B): $P$ is not finitely generated.

We have $P^{2} \neq(0)$, by assumption. Hence there exist $a_{1}, a_{2} \in P$ such that $a_{1} \neq a_{2}$ and $a_{1} a_{2} \neq 0$. Suppose that $P$ is not a $B$-prime of $(0)$ in $R$. Then, as $\left((0):_{R} a_{1} a_{2}\right) \subseteq Z(R)=P$, it follows that $P \not \subset\left((0):_{R} a_{1} a_{2}\right)$. As $R a_{1}+R a_{2} \subseteq P$ and since $P$ is not finitely generated, it follows that $P \not \subset R a_{1}+R a_{2}$. Hence $P \not \subset\left(R a_{1}+R a_{2}\right) \cup\left((0):_{R} a_{1} a_{2}\right)$. Hence there exists $a_{3} \in$ $P \backslash\left(\left(R a_{1}+R a_{2}\right) \cup\left((0):_{R} a_{1} a_{2}\right)\right)$. Thus $a_{1}, a_{2}, a_{3} \in P$ are distinct and $a_{1} a_{2} a_{3} \neq 0$. Let $k$ be any positive integer with $k \geq 3$. Assume that there exists a subset $\left\{a_{1}, a_{2}, a_{3}, \ldots, a_{k}\right\}$ of $P$ with $a_{1} a_{2} a_{3} \cdots a_{k} \neq 0$. Observe that $P \not \subset R a_{1}+R a_{2}+R a_{3}+\cdots+R a_{k}$ and $P \not \subset\left((0):_{R} a_{1} a_{2} a_{3} \cdots a_{k}\right)$. Hence there exists $a_{k+1} \in P \backslash\left(\left(R a_{1}+\cdots+R a_{k}\right) \cup\left((0):_{R} a_{1} a_{2} a_{3} \cdots a_{k}\right)\right)$. This shows that if $P$ is not finitely generated and if $P$ is not a $B$-prime of (0) in $R$, then there exists an infinite subset $\left\{a_{i} \mid i=1,2,3, \ldots\right\}$ of $P$ such that the product $a_{1} \cdots a_{k} \neq 0$ for $k=1,2,3, \ldots$. This implies that the subgraph of $(\Gamma(R))^{c}$ induced on $\left\{a_{i} \mid i=1,2,3, \ldots\right\}$ is an infinite clique.

This contradicts the hypothesis that $(\Gamma(R))^{c}$ does not contain any infinite clique. Hence $P$ is a $B$-prime of $(0)$ in $R$.

(iv) We obtain from (ii) that $R / P$ is finite. Since any finite integral domain is a field, it follows that $P$ is a maximal ideal of $R$. By (i), $P=\operatorname{nil}(R)$. Hence we obtain that $P$ is the only prime ideal of $R$. We now verify that $R$ satisfies d. c. c. on principal ideals. Suppose that $R$ does not satisfy d. c. c. on principal ideals. Then there exist nonzero elements $x_{i} \in P$ for $i=1,2,3, \ldots$ such that $R x_{1} \supset R x_{2} \supset R x_{3} \supset \ldots$. Note that there exist $a_{i} \in P$ for $i=1,2,3, \ldots$ such that $x_{i+1}=a_{i} x_{i}$. Hence

$$
x_{k+1}=\left(a_{k} \cdots a_{1}\right) x_{1}, \quad \forall k \geq 1 .
$$

Since $x_{k} \neq 0$ for $k=1,2,3, \ldots$, it follows that the elements $a_{k} \in P(k=1,2,3, \ldots)$ satisfy $a_{i} a_{j} \neq 0$ for all distinct $i, j \in\{1,2,3, \ldots\}$. As each element of $P$ is nilpotent, it follows from (I) and the fact that $x_{k} \neq 0$ for $k=1,2,3, \ldots$ that there exist positive integers $k_{1}<k_{2}<k_{3}<\cdots$ such that for all distinct $i, j \in\left\{k_{1}, k_{2}, \ldots\right\}, a_{i} \neq a_{j}$. Let $A=\left\{k_{i} \mid i=1,2,3, \ldots\right\}$. Observe that the subgraph of $(\Gamma(R))^{c}$ induced on $\left\{a_{i} \mid i \in A\right\}$ is an infinite clique. This contradicts the assumption that $(\Gamma(R))^{c}$ does not contain any infinite clique. Hence it follows that $R$ satisfies d. c. c. on principal ideals.

Let $R$ and $P$ be as in Lemma 4.14. Suppose that $P^{2} \neq(0)$. I do not know any necessary and sufficient condition in order that $(\Gamma(R))^{c}$ does not contain any infinite clique. However, the following proposition shows that if the ring $R$ is Noetherian, then $(\Gamma(R))^{c}$ does not contain any infinite clique if and only if $R$ is finite. 
Proposition 4.15. Let $R$ be a Noetherian ring which is not an integral domain. Suppose that $R$ has only one maximal $N$-prime of $(0)$ and let it be $P$. If $P^{2} \neq(0)$, then the following conditions are equivalent.

(i) $\omega\left((\Gamma(R))^{c}\right)$ is finite.

(ii) $(\Gamma(R))^{c}$ does not contain any infinite clique.

(iii) $R$ is finite.

Proof. (i) $\Rightarrow$ (ii) This is clear.

(ii) $\Rightarrow$ (iii) We know from Lemma 4.14 that $P=$ the nilradical of $R$ and $R / P$ is finite. Now by hypothesis, $R$ is a Noetherian ring. Hence $P$ is finitely generated. Therefore, $P^{n}=(0)$ for some $n \geq 1$. Since $P^{2} \neq(0)$, it follows that $n \geq 3$. Observe that for each integer $k, 0 \leq k \leq$ $n-1, P^{k} / P^{k+1}$ is a finite-dimensional vector space over the finite field $R / P$. Hence it follows that $P^{k} / P^{k+1}$ is finite for $k=0,1,2, \ldots, n-1$. Now $P^{n-1}, P^{n-2} / P^{n-1}$ are finite, and hence it follows that $P^{n-2}$ is finite. Proceeding in this way, we obtain that $P$ is finite. Thus $P$ and $R / P$ are finite. Hence we obtain that $R$ is finite.

(iii) $\Rightarrow$ (i) This is clear.

Recall that a commutative ring $R$ with identity is said to be a chained ring if the principal ideals of $R$ are linearly ordered under inclusion (equivalently, the ideals of $R$ are linearly ordered under inclusion).

Let $R$ be a chained ring which is not an integral domain. Then, it is clear that $R$ must have exactly one maximal $N$-prime of (0). If $P$ is the only maximal $N$-prime of $(0)$ and if $P^{2} \neq(0)$, then the following proposition characterizes when $(\Gamma(R))^{c}$ can admit infinite cliques.

Proposition 4.16. Let $R$ be a commutative ring with identity which is not an integral domain. Suppose that $R$ is a chained ring and moreover, there exist $x, y \in Z(R)^{*}$ with $x \neq y$ such that $x y \neq 0$. Then the following conditions are equivalent.

(i) $(\Gamma(R))^{c}$ does not admit any infinite clique.

(ii) $R$ is finite.

(iii) $\omega\left((\Gamma(R))^{c}\right)$ is finite.

Proof. (i) $\Rightarrow$ (ii) Since the ideals of $R$ are linearly ordered under inclusion, it follows that $R$ admits exactly one maximal $N$-prime of (0). Let $P$ be the unique maximal $N$-prime of $(0)$ in $R$. We are assuming that $(\Gamma(R))^{c}$ does not admit any infinite clique. So, we obtain from Lemma 4.14(i) that $P=$ the nilradical of $R$. Note that $Z(R)=P$. Let $N(R)=\left\{x \in P \mid x^{2}=0\right\}$. It is known that for any $x, y \in P \backslash N(R), x y \neq 0$ [6, Lemma 4.2(3)]. Since $(\Gamma(R))^{c}$ does not admit any infinite clique, it follows that $P \backslash N(R)$ is finite. Now by the assumption that $R$ is a chained ring and there exist $x, y \in Z(R)^{*}$ with $x \neq y$ such that $x y \neq 0$, it follows that $Z(R) \backslash N(R)$ is non-empty. Let $P \backslash N(R)=\left\{x_{1}, \ldots, x_{m}\right\}$. Since each element of $P$ is nilpotent, it follows that there exist $t>2$ such that $p^{t}=0$ for each $p \in P$. As $R$ is a chained ring, we obtain that $P^{t}=(0)$. Moreover, it follows from Lemma 4.14(ii) that $R / P$ is finite. Hence $P$ is a maximal ideal of $R$ and since $R$ is a chained ring, it follows that $R$ is quasilocal with $P$ as its unique maximal ideal. As $P$ is nilpotent and $P \neq(0)$, it follows that $P \neq P^{2}$. Now $R$ is a chained 
ring with $P$ as its unique maximal ideal satisfying the further condition that $P \neq P^{2}$. In such a case it is well known that $P=R p$ for any $p \in P \backslash P^{2}$. Using the same reasoning as in the proof of (ii) $\Rightarrow$ (iii) of Proposition 4.15, it now follows that $R$ is finite.

(ii) $\Rightarrow$ (iii) and (iii) $\Rightarrow$ (i) are clear.

The following remark determines $\omega\left((\Gamma(R))^{c}\right)$ for any finite chained ring which is not an integral domain.

Remark 4.17. Let $R$ be a finite chained ring which is not an integral domain. Let $P$ denote the unique maximal ideal of $R$. Suppose that $P^{2} \neq(0)$. Let $n \geq 3$ be least with the property that $P^{n}=(0)$. Then the following hold.

(i) $\omega\left((\Gamma(R))^{c}\right)=\left|P \backslash P^{n / 2}\right|+1$ if $n$ is even.

(ii) $\omega\left((\Gamma(R))^{c}\right)=\left|P \backslash P^{(n+1) / 2}\right|$ if $n$ is odd.

Proof. As is already observed in the proof of (i) $\Rightarrow$ (ii) of Proposition $4.16, P=R p$ for any $p \in$ $P \backslash P^{2}$.

(i) Suppose that $n$ is even and let $n=2 m$. By the choice of $n, p^{n-1} \neq 0$. If $a \in P \backslash P^{m}, b \in$ $P \backslash P^{m}$, then $a=u p^{t}$ for some $t, 1 \leq t<m$ and $a$ unit $u$ in $R$ and $b=v p^{s}$ for some $s, 1 \leq s<m$ and $a$ unit $v$ in $R$. Hence $a b=u v p^{t+s} \neq 0$, since $t+s<2 m=n$ and $p^{n-1} \neq 0$. Moreover, observe that $p^{m} x \neq 0$ for any $x \in P \backslash P^{m}$. Hence the subgraph of $(\Gamma(R))^{c}$ induced on $\left(P \backslash P^{m}\right) \cup\left\{p^{m}\right\}$ is a clique. This implies that $\omega\left((\Gamma(R))^{c}\right) \geq\left|P \backslash P^{m}\right|+1$. Let $A \subseteq Z(R)^{*}=P \backslash\{0\}$ be such that the subgraph of $(\Gamma(R))^{c}$ induced on $A$ is a clique. Let $|A|=t$. Since $R$ is a chained ring, it is possible to find $a \in A$ such that $R a \subseteq R b$ for each $b \in A$. Hence $R a b \subseteq R b^{2}$. Since $a b \neq 0$ for each $b \in A \backslash\{a\}$, we obtain that $b^{2} \neq 0$ for each $b \in A \backslash\{a\}$. As $P^{n}=P^{2 m}=(0)$, it follows that $A \backslash\{a\} \subseteq P \backslash P^{m}$. Thus $A=\{a\} \cup(A \backslash\{a\}) \subseteq\{a\} \cup\left(P \backslash P^{m}\right)$ and so $|A| \leq\left|P \backslash P^{m}\right|+1$. This proves that $\omega\left((\Gamma(R))^{c}\right) \leq\left|P \backslash P^{m}\right|+1$. Hence $\omega\left((\Gamma(R))^{c}\right)=\left|P \backslash P^{m}\right|+1$.

(ii) Suppose that $n$ is odd and let $n=2 k+1$ for some $k \geq 1$. In this case, we verify that $\omega\left((\Gamma(R))^{c}\right)=\left|P \backslash P^{k+1}\right|$ where $k=(n-1) / 2$. It is clear that for any $x, y \in P \backslash P^{k+1}, \quad x y \neq 0$. This shows that the subgraph of $(\Gamma(R))^{c}$ induced on $P \backslash P^{k+1}$ is a clique. Hence we obtain that $\omega\left((\Gamma(R))^{c}\right) \geq\left|P \backslash P^{k+1}\right|$. Let $A \subseteq Z(R)^{*}=P \backslash\{0\}$ be such that the subgraph of $(\Gamma(R))^{c}$ induced on $A$ is a clique. We assert that $|A| \leq\left|P \backslash P^{k+1}\right|$. Let $a \in A$ be such that $R a \subseteq R b$ for each $b \in A$. Now, it follows as in the proof of (i) that $b^{2} \neq 0$ for each $b \in A \backslash\{a\}$. Since $P^{2 k+1}=(0)$, it follows that $A \backslash\{a\} \subseteq P \backslash P^{k+1}$. If there exists at least one $b \in A \backslash\{a\}$ such that $b \in P^{k}$, then, as $a b \neq 0$, it follows that $a \notin P^{k+1}$ and so we obtain that $A \subseteq P \backslash P^{k+1}$. Hence it follows that $|A| \leq\left|P \backslash P^{k+1}\right|$. Otherwise, we obtain that $A \backslash\{a\} \subseteq P \backslash P^{k}$. Note that $A=\{a\} \cup(A \backslash\{a\}) \subseteq\{a\} \cup\left(P \backslash P^{k}\right)$ and so $|A| \leq 1+\left|P \backslash P^{k}\right| \leq\left|P^{k} \backslash P^{k+1}\right|+\left|P \backslash P^{k}\right|=\left|P \backslash P^{k+1}\right|$. This proves that $\omega\left((\Gamma(R))^{c}\right) \leq\left|P \backslash P^{k+1}\right|$. Hence we obtain that

$$
\omega\left((\Gamma(R))^{c}\right)=\left|P \backslash P^{k+1}\right|=\left|P \backslash P^{(n+1) / 2}\right| .
$$

We next mention an example to illustrate that in Remark 4.17, one cannot drop the assumption that $R$ is a chained ring.

Example 4.18. Let $F=Z / 2 Z$ be the field containing exactly two elements. It is convenient to denote $F$ simply by $F=\{0,1\}$. Let $p(x)=x^{2}+x+1 \in F[x]$ where $F[x]$ is the polynomial ring in one variable over $F$. Note that $p(x)$ is irreducible over $F$. Let $K$ be the splitting field of $p(x)$ over $F$. Let $\alpha \in K$ be a root of $p(x)$. It is clear that $K=F(\alpha)$ and $[K: F]=2$. Indeed, 
$\{1, \alpha\}$ is a basis of $K$ as a vector space over $F$. Moreover, $K=\left\{0,1, \alpha, \alpha^{2}=-\alpha-1=\alpha+1\right\}$. Let $T=K[[x]]$ be the power series ring in one variable over $K$. Note that $T=K+M$ where $M=x K[[x]]=x T$. Let $S=F+M$. Consider the ring $R=S / x^{2} S$. Let us denote $x^{2} S$ by $\mathrm{I}$. Observe that $R=\left\{\left(a+(b+c \alpha) x+(d+e \alpha) x^{2}\right)+I \mid a, b, c, d, e \in F\right\}$. Note that $R$ is a finite local ring with unique maximal ideal $N=M / I$ satisfying $N^{3}=$ the zero-ideal of $R$, but $N^{2}$ is nonzero. We have $\alpha^{2}=1+\alpha$ and thus $N=\left\{0+I, \quad x+I, \quad \alpha x+I, \quad \alpha^{2} x+I, \alpha x^{2}+I,\left(x+\alpha x^{2}\right)+I\right.$, $\left.\left(\alpha x+\alpha x^{2}\right)+I,\left(\alpha^{2} x+\alpha x^{2}\right)+I\right\}$. Observe that $Z(R)=N$. Now $N \backslash N^{2}=\{x+I, \alpha x+I$, $\left.\alpha^{2} x+I,\left(x+\alpha x^{2}\right)+I,\left(\alpha x+\alpha x^{2}\right)+I,\left(\alpha^{2} x+\alpha x^{2}\right)+I\right\}$. Thus $\left|N \backslash N^{2}\right|=6$. We assert that $\omega\left((\Gamma(R))^{c}\right)=3$. Since $N^{3}=$ the zero-ideal of $R$, it follows that the vertex set of any clique in $(\Gamma(R))^{c}$ must be a subset of

$$
N \backslash N^{2}
$$

As $\alpha \notin F, \alpha x^{2} \notin I=x^{2} S$, and $\alpha^{2} x^{2}=(1+\alpha) x^{2} \notin I$. Hence the subgraph of $(\Gamma(R))^{c}$ induced on $\left\{x+I, \alpha x+I,\left(\alpha x+\alpha x^{2}\right)+I\right\}$ is a clique. This shows that $\omega\left((\Gamma(R))^{c}\right) \geq 3$. Observe that

$$
(x+I)\left(\left(x+\alpha x^{2}\right)+I\right)=x^{2}+I=0+I .
$$

We have $\alpha^{3}=\alpha \alpha^{2}=\alpha(1+\alpha)=\alpha+\alpha^{2}=\alpha+(1+\alpha)=1$. Note that

$$
(\alpha x+I)\left(\alpha^{2} x+I\right)=x^{2}+I=0+I
$$

Moreover,

$$
\left(\left(\alpha x+\alpha x^{2}\right)+I\right)\left(\left(\alpha^{2} x+\alpha x^{2}\right)+I\right)=x^{2}+I=0+I .
$$

It is clear from (4.7), (4.8), (4.9), and (4.10) that if $A \subseteq Z(R)^{*}$ is such that the subgraph of $(\Gamma(R))^{c}$ induced on $A$ is a clique, then $A$ can contain at most 3 elements. Hence we obtain that $\omega\left((\Gamma(R))^{c}\right) \leq 3$. Thus $\omega\left((\Gamma(R))^{c}\right)=3<\left|N \backslash N^{2}\right|=6$.

Let $T$ and $S$ be as above. Let $R_{1}=S / x^{3} S$. Note that $R_{1}$ is a finite local ring with $N_{1}=$ $M / x^{3} S$ as its unique maximal ideal. Thus $Z\left(R_{1}\right)=N_{1}$. Moreover, note that $\left(N_{1}\right)^{4}$ is the zeroideal of $R_{1}$ but $\left(N_{1}\right)^{3}$ is nonzero. It is convenient to denote the ideal $x^{3} S$ of $S$ by $J$. Note that $\alpha^{2}=\alpha+1$. We assert that $\omega\left(\left(\Gamma\left(R_{1}\right)\right)^{c}\right)=\left|N_{1} \backslash\left(N_{1}\right)^{2}\right|$. Since $\left(N_{1}\right)^{4}=(0)$, it follows that if $A$ is any subset of $Z\left(R_{1}\right)^{*}=N_{1} \backslash\{0+J\}$ such that the subgraph of $\left(\Gamma\left(R_{1}\right)\right)^{c}$ induced on $A$ is a clique, then $A$ can admit at most one element of $\left(N_{1}\right)^{2}$. We claim that $|A| \leq\left|N_{1} \backslash\left(N_{1}\right)^{2}\right|$. This is clear if $A$ does not contain any element of $\left(N_{1}\right)^{2}$. Suppose that $A$ has an element of $\left(N_{1}\right)^{2}$. In such a case, we verify that $A$ cannot contain all the elements of $N_{1} \backslash\left(N_{1}\right)^{2}$. Let $a \in A$ be such that $a \in\left(N_{1}\right)^{2}$. Note that $a=\left(\beta x^{2}+\gamma x^{3}\right)+J$ for some $\beta, \gamma \in K$. We consider two cases.

Case i. $\beta=0$. In such a case, the element $a=\gamma x^{3}+J$ annihilates each element of $N_{1}$ and hence it annihilates each element of $N_{1} \backslash\left(N_{1}\right)^{2}$.

Case ii. $\beta \neq 0$. Observe that $\beta=u+v \alpha$ for some $u, v \in F$ with at least one of $u, v$ is different from 0 . Thus $a \in\left\{\left(x^{2}+\gamma x^{3}\right)+J,\left(\alpha x^{2}+\gamma x^{3}\right)+J,\left(\alpha^{2} x^{2}+\gamma x^{3}\right)+J\right\}$. Note that $\left(x^{2}+\gamma x^{3}\right)+J$ annihilates 
the element $x+J \in N_{1} \backslash\left(N_{1}\right)^{2}$. The element $\left(\alpha x^{2}+\gamma x^{3}\right)+J$ annihilates $\alpha^{2} x+J \in N_{1} \backslash\left(N_{1}\right)^{2}$ and the element $\left(\alpha^{2} x^{2}+\gamma x^{3}\right)+J$ annihilates $\alpha x+J \in N_{1} \backslash\left(N_{1}\right)^{2}$.

This shows that if $A$ contains an element of $\left(N_{1}\right)^{2}$, then that element annihilates at least one element of $N_{1} \backslash\left(N_{1}\right)^{2}$. Since the subgraph of $\left(\Gamma\left(R_{1}\right)\right)^{c}$ induced on $A$ is a clique, it follows that $A$ cannot contain all the elements of $N_{1} \backslash\left(N_{1}\right)^{2}$. This proves that $|A| \leq\left|N_{1} \backslash\left(N_{1}\right)^{2}\right|$ and hence we obtain that $\omega\left(\left(\Gamma\left(R_{1}\right)\right)^{c}\right) \leq\left|N_{1} \backslash\left(N_{1}\right)^{2}\right|$.

We next claim that the subgraph of $\left(\Gamma\left(R_{1}\right)\right)^{c}$ induced on $N_{1} \backslash\left(N_{1}\right)^{2}$ is a clique. Let $a$, $b \in N_{1} \backslash\left(N_{1}\right)^{2}$. Note that $a=\left(\beta_{1} x+\beta_{2} x^{2}+\beta_{3} x^{3}\right)+J$ and $b=\left(\gamma_{1} x+\gamma_{2} x^{2}+\gamma_{3} x^{3}\right)+J$ for some $\beta_{i}, \gamma_{i} \in K$ (for $i=1,2,3$ ) with $\beta_{1}, \gamma_{1} \in K \backslash\{0\}$. Note that $a b=\beta_{1} \gamma_{1} x^{2}+w+J$ for some $w \in x^{3} K[[x]]$. Since, $J=x^{3} S \subseteq x^{3} K[[x]]$ and as $\beta_{1} \gamma_{1} x^{2} \notin x^{3} K[[x]]$, it follows that $a b$ is a nonzero element of $R_{1}$. This proves that the subgraph of $\left(\Gamma\left(R_{1}\right)\right)^{c}$ induced on $N_{1} \backslash\left(N_{1}\right)^{2}$ is a clique. Hence we obtain that $\omega\left(\left(\Gamma\left(R_{1}\right)\right)^{c}\right) \geq\left|N_{1} \backslash\left(N_{1}\right)^{2}\right|$ and so $\omega\left(\left(\Gamma\left(R_{1}\right)\right)^{c}\right)=\left|N_{1} \backslash\left(N_{1}\right)^{2}\right|$.

Let $R$ be a commutative ring with identity which is not an integral domain. Suppose that $R$ has exactly one maximal $N$-prime of $(0)$ and let it be $P$. If $(\Gamma(R))^{c}$ does not admit any infinite clique, then it was shown in Lemma 4.14 that each element of $P$ is nilpotent. The following lemma describes the elements of $P$ under the hypothesis that $\omega\left((\Gamma(R))^{c}\right)$ is finite.

Lemma 4.19. Let $R$ be a commutative ring with identity which is not an integral domain. Suppose that $P$ is the only maximal $N$-prime of $(0)$ in $R$. If $\omega\left((\Gamma(R))^{c}\right)$ is finite, then for any $x \in P, x^{m}=0$ where $m=\omega\left((\Gamma(R))^{c}\right)+1$.

Proof. By hypothesis, $\omega\left((\Gamma(R))^{c}\right)$ is finite. Let $x \in P$. We assert that $x^{m}=0$ where $m=$ $\omega\left((\Gamma(R))^{c}\right)+1$. Suppose that $x^{m} \neq 0$. For each $k \in\{1,2, \ldots, m\}$, let $y_{k}=x+\cdots+x^{k}$. Note that $Z(R)=P$ and $r_{1}=1 \notin P=Z(R)$ and for $2 \leq k \leq m, r_{k}=1+\cdots+x^{k-1} \notin P=Z(R)$ and moreover, $y_{k}=x r_{k}$ for $k=1,2, \ldots, m$. Since $x^{2} \neq 0$ and $r_{i} r_{j} \notin Z(R)$, it follows that $y_{i} y_{j} \neq 0$ for all $i, j \in\{1,2, \ldots, m\}$. Let $i, j \in\{1,2, \ldots, m\}$ with $i \neq j$. We claim that $y_{i} \neq y_{j}$. Suppose that $y_{i}=y_{j}$. We may assume without loss of generality that $i<j$. Then $y_{i}=y_{j}$ implies that $x^{i+1}\left(1+\cdots+x^{j-(i+1)}\right)=0$. As $1+\cdots+x^{j-(i+1)} \notin Z(R)$, it follows that $x^{i+1}=0$, and this is not possible since by assumption, $x^{m} \neq 0$. Hence $y_{i} \neq y_{j}$ for all distinct $i, j \in\{1,2, \ldots, m\}$ and moreover, $y_{i} y_{j} \neq 0$. Hence we obtain that the subgraph of $(\Gamma(R))^{c}$ induced on $\left\{y_{k} \mid k=1,2, \ldots, m\right\}$ is a clique. This implies that $\omega\left((\Gamma(R))^{c}\right) \geq m$. This is impossible since $m=\omega\left((\Gamma(R))^{c}\right)+1$. Hence we obtain that $x^{m}=0$ for any $x \in P$.

The next remark provides examples of rings $R$ for which $\omega\left((\Gamma(R))^{c}\right)=\infty$.

Remark 4.20. We remark here that Lemma 4.19 is motivated by [3, Theorem 3.4]. Let $R$ be a commutative ring with identity which is not reduced. Let $x \in \operatorname{nil}(R)$. Recall that by the index of nilpotence of $x$, we mean the least positive integer $n$ such that $x^{n}=0$. Suppose that $x \in \operatorname{nil}(R)$ with $x \neq 0$. Let $n$ be the index of nilpotence of $x$. Using the fact that the sum of a nilpotent element and a unit in any ring is a unit, it can be shown as in the proof of Lemma 4.19 that $\left\{y_{k}=x+\cdots+x^{k} \mid k=1, \ldots, n-1\right\}$ is a clique in $(\Gamma(R))^{c}$. Hence it follows that $\omega\left((\Gamma(R))^{c}\right) \geq n-1$. Thus if a commutative ring $R$ with identity is such that there is no bound on the index of nilpotence of nilpotent elements of $R$, then it follows that $\omega\left((\Gamma(R))^{c}\right)=\infty$.

Let $R$ and $P$ be as in Lemma 4.19. If $\omega\left((\Gamma(R))^{c}\right)$ is finite, then with the help of Lemma 4.19 , we prove in the following proposition that $P$ is nilpotent. 
Proposition 4.21. Let $R$ be a commutative ring with identity which is not an integral domain. Suppose that $R$ has only one maximal $N$-prime of $(0)$ and let it be $P$. If $\omega\left((\Gamma(R))^{c}\right)$ is finite, then $P$ is nilpotent.

Proof. Let $m=\omega\left((\Gamma(R))^{c}\right)+1$. We claim that $P^{n}=(0)$ with $n=(m-1)^{2}+1=\left(\omega\left((\Gamma(R))^{c}\right)\right)^{2}+1$. Suppose that $P^{n} \neq(0)$. Then there exist $x_{k} \in P$ for $k=1,2, \ldots, n$ such that $x_{1} x_{2} \cdot \cdots x_{n} \neq 0$. Let $s$ be the number of distinct elements among $x_{1}, x_{2}, \ldots, x_{n}$. Note that we may assume without loss of generality that $x_{1}, \ldots, x_{s}$ are the distinct elements among $x_{1}, x_{2}, \ldots, x_{n}$. Let $j \in\{1, \ldots, s\}$, and let $A_{j}=\left\{k \in\{1,2, \ldots, n\} \mid x_{k}=x_{j}\right\}$. Let $\left|A_{j}\right|=n_{j}$ for $j=1, \ldots, s$. Note that $\{1,2, \ldots, n\}=\bigcup_{\mathrm{j}=1}^{\mathrm{s}} A_{j}$, and moreover, $A_{i} \cap A_{j}=\emptyset$ for all distinct $i, j \in\{1, \ldots, s\}$. Hence we obtain that $n=\sum_{j=1}^{s}\left|A_{j}\right|=n_{1}+\cdots+n_{s}$. Since $x_{1} x_{2} \cdots x_{n} \neq 0$, it follows that $x_{1} \cdots x_{s} \neq 0$, and moreover, for each $j=1, \ldots, s$, the product of $n_{j}$ factors of $x_{j}$ is different from 0 . We know from Lemma 4.19 that $x^{m}=0$ for any $x \in P$. Hence it follows that $n_{j} \leq m-1$ for $j=1, \ldots, s$. Furthermore, observe that the subgraph of $(\Gamma(R))^{c}$ induced on $\left\{x_{1}, \ldots, x_{s}\right\}$ is a clique and so $s \leq \omega\left((\Gamma(R))^{c}\right)=m-1$. Thus we obtain that $(m-1)^{2}+1=n=\sum_{j=1}^{s} n_{j} \leq s(m-1) \leq$ $(m-1)(m-1)$. This is impossible. Hence it follows that $P^{n}=(0)$ with $n=(m-1)^{2}+1$.

We conclude this note with the following example of an infinite ring $R$ such that $R$ has exactly one maximal $N$-prime of $(0)$ satisfying the property that $\omega\left((\Gamma(R))^{c}\right)=3$, thereby illustrating that Proposition 4.15 need not hold for non-Noetherian rings.

Example 4.22. Let $T=Z_{2}\left[x_{1}, x_{2}, x_{3}, \ldots\right]$ be the polynomial ring in an infinite number of variables over $Z_{2}$. Let $I$ be the ideal of $T$ generated by $\left\{x_{k}^{2} \mid k=1,2,3, \ldots\right\} \cup\left\{x_{1} x_{k} \mid k=\right.$ $3,4,5, \ldots\} \cup\left\{x_{i} x_{j} \mid i, j \in \mathbf{N}, 2 \leq i<j\right\}$. Let $R=T / I$. Let $M$ be the ideal of $T$ generated by $\left\{x_{k} \mid k=1,2,3, \ldots\right\}$. Observe that $P=M / I$ is the only prime ideal of $R$. Thus $Z(R)=P$. It is clear that $R$ is infinite. Note that $x_{1}+I-\left(x_{1}+x_{2}\right)+I-x_{2}+I-x_{1}+I$ is a cycle of length 3 in $(\Gamma(R))^{c}$ and hence we obtain that $\omega\left((\Gamma(R))^{c}\right) \geq 3$. We next verify that $\omega\left((\Gamma(R))^{c}\right) \leq 3$. Let $A \subseteq Z(R)^{*}$ be such that the subgraph of $(\Gamma(R))^{c}$ induced on $A$ is a clique. We assert that $A$ can contain at most 3 elements. Suppose that $A$ contains more than 3 elements. Note that for any $z, w \in A$ with $z \neq w, z w \neq 0+I$.

Let $z, w \in Z(R)^{*}$. Observe that $z=f_{1}\left(x_{1}, x_{2}, \ldots, x_{m}\right)+I, w=f_{2}\left(x_{1}, x_{2}, \ldots, x_{m}\right)+I$, for some $m \geq 2$ and for some $f_{1}\left(x_{1}, \ldots, x_{m}\right), f_{2}\left(x_{1}, \ldots, x_{m}\right) \in Z_{2} x_{1}+Z_{2} x_{2}+\cdots+Z_{2} x_{m}+Z_{2} x_{1} x_{2}$. It is clear that $z w=0+I$ if the coefficient of $x_{2}$ is 0 in

$$
f_{i}\left(x_{1}, \ldots, x_{m}\right) \text { for } i=1,2 \text {. }
$$

We are assuming that $A$ contains at least 4 elements. Let $\left\{z_{1}, z_{2}, z_{3}, z_{4}\right\} \subseteq A$. Let $z_{k}=$ $g_{k}\left(x_{1}, x_{2}, \ldots, x_{m}\right)+I$, for some $m \geq 2$ and for some $g_{k}\left(x_{1}, x_{2}, \ldots, x_{m}\right) \in Z_{2} x_{1}+Z_{2} x_{2}+$. $\cdot+Z_{2} x_{m}+Z_{2} x_{1} x_{2}$ for $k=1,2,3,4$. Since $z_{i} z_{j} \neq 0+I$ for all distinct $i, j \in\{1,2,3,4\}$, it follows from (4.11) that the coefficient of $x_{2}$ must be 1 for all $g_{k}\left(x_{1}, x_{2}, \ldots, x_{m}\right)(k=1,2,3,4)$ except possibly one value of $k$. We may assume without loss of generality that $g_{k}\left(x_{1}, x_{2}, \ldots, x_{m}\right)=$ $a_{k 1} x_{1}+x_{2}+\cdots+a_{k m} x_{m}+a_{k 12} x_{1} x_{2}$ for $k=1,2,3$ where $a_{k 1}, a_{k 3}, \ldots, a_{k m}, a_{k 12} \in Z_{2}$. Since $z_{1} z_{2} \neq 0+I$, it follows that exactly one among $a_{11}, a_{21}$ must be 1 . We may assume without loss of generality that $a_{11}=1$ and $a_{21}=0$. Now either $a_{31}=0$ or $a_{31}=1$. If $a_{31}=0$, then we arrive at $z_{2} z_{3}=0+I$, which is impossible. If $a_{31}=1$, then we obtain that $z_{1} z_{3}=0+I$, and this is also impossible. 
ISRN Algebra

This proves that if $A \subseteq Z(R)^{*}$ is such that the subgraph of $(\Gamma(R))^{c}$ induced on $A$ is a clique, then $|A| \leq 3$. Hence we obtain that $\omega\left((\Gamma(R))^{c}\right) \leq 3$. This proves that $\omega\left((\Gamma(R))^{c}\right)=3$.

\section{Acknowledgments}

The author is very much thankful to the academic editors Professor David F. Anderson, Professor Vesselin Drensky, and Professor Dolors Herbera for their useful and valuable suggestions.

\section{References}

[1] D. F. Anderson and P. S. Livingston, "The zero-divisor graph of a commutative ring," Journal of Algebra, vol. 217, no. 2, pp. 434-447, 1999.

[2] D. D. Anderson and M. Naseer, "Beck's coloring of a commutative ring," Journal of Algebra, vol. 159, no. 2, pp. 500-514, 1993.

[3] D. F. Anderson, A. Frazier, A. Lauve, and P. S. Livingston, "The zero-divisor graph of a commutative ring. II," in Ideal theoretic methods in commutative algebra, vol. 220 of Lecture Notes in Pure and Applied Mathematics, pp. 61-72, Dekker, New York, NY, USA, 2001.

[4] D. F. Anderson, R. Levy, and J. Shapiro, "Zero-divisor graphs, von Neumann regular rings, and Boolean algebras," Journal of Pure and Applied Algebra, vol. 180, no. 3, pp. 221-241, 2003.

[5] D. F. Anderson and S. B. Mulay, "On the diameter and girth of a zero-divisor graph," Journal of Pure and Applied Algebra, vol. 210, no. 2, pp. 543-550, 2007.

[6] D. F. Anderson and A. Badawi, "On the zero-divisor graph of a ring," Communications in Algebra, vol. 36, no. 8, pp. 3073-3092, 2008

[7] M. Axtell, J. Coykendall, and J. Stickles, "Zero-divisor graphs of polynomials and power series over commutative rings," Communications in Algebra, vol. 33, no. 6, pp. 2043-2050, 2005.

[8] F. Azarpanah and M. Motamedi, "Zero-divisor graph of C(X)," Acta Mathematica Hungarica, vol. 108, no. 1-2, pp. 25-36, 2005.

[9] I. Beck, "Coloring of commutative rings," Journal of Algebra, vol. 116, no. 1, pp. 208-226, 1988.

[10] R. Levy and J. Shapiro, "The zero-divisor graph of von Neumann regular rings," Communications in Algebra, vol. 30, no. 2, pp. 745-750, 2002.

[11] T. G. Lucas, "The diameter of a zero divisor graph," Journal of Algebra, vol. 301, no. 1, pp. 174-193, 2006.

[12] S. B. Mulay, "Cycles and symmetries of zero-divisors," Communications in Algebra, vol. 30, no. 7, pp. 3533-3558, 2002.

[13] S. P. Redmond, "Central sets and radii of the zero-divisor graphs of commutative rings," Communications in Algebra, vol. 34, no. 7, pp. 2389-2401, 2006.

[14] K. Samei, "The zero-divisor graph of a reduced ring," Journal of Pure and Applied Algebra, vol. 209, no. 3, pp. 813-821, 2007.

[15] R. Balakrishnan and K. Ranganathan, A Textbook of Graph Theory, Universitext, Springer, New York, NY, USA, 2000.

[16] W. Heinzer and J. Ohm, "On the Noetherian-like rings of E. G. Evans," Proceedings of the American Mathematical Society, vol. 34, pp. 73-74, 1972.

[17] W. Heinzer and J. Ohm, "Locally noetherian commutative rings," Transactions of the American Mathematical Society, vol. 158, pp. 273-284, 1971.

[18] S. Visweswaran, "Some results on the complement of the zero-divisor graph of a commutative ring," Journal of Algebra and its Applications, vol. 10, no. 3, pp. 573-595, 2011.

[19] T. W. Hungerford, Algebra, vol. 73 of Graduate Texts in Mathematics, Springer, New York, NY, USA, 1980.

[20] N. Ganesan, "Properties of rings with a finite number of zero divisors," Mathematische Annalen, vol. 157, pp. 215-218, 1964.

[21] M. F. Atiyah and I. G. Macdonald, Introduction to Commutative Algebra, Addison-Wesley Publishing Company, Reading, Mass, USA, 1969.

[22] I. Kaplansky, Commutative Rings, The University of Chicago Press, Chicago, Ill, USA, 1974.

[23] R. Gilmer, Multiplicative ideal theory, Dekker, New York, NY, USA, 1972.

[24] S. Visweswaran, "ACCR pairs," Journal of Pure and Applied Algebra, vol. 81, no. 3, pp. 313-334, 1992. 


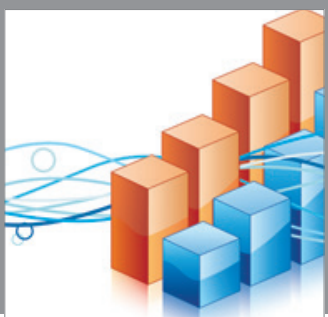

Advances in

Operations Research

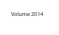

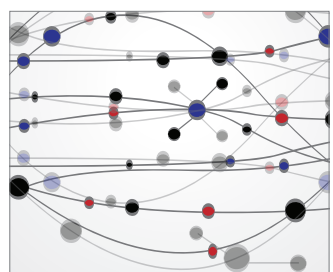

\section{The Scientific} World Journal
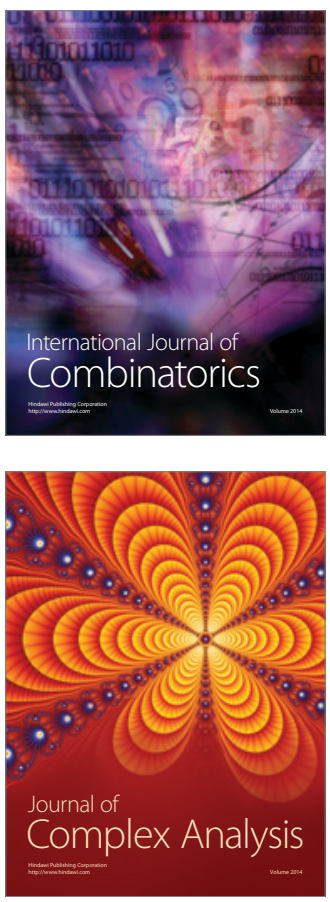

International Journal of

Mathematics and

Mathematical

Sciences
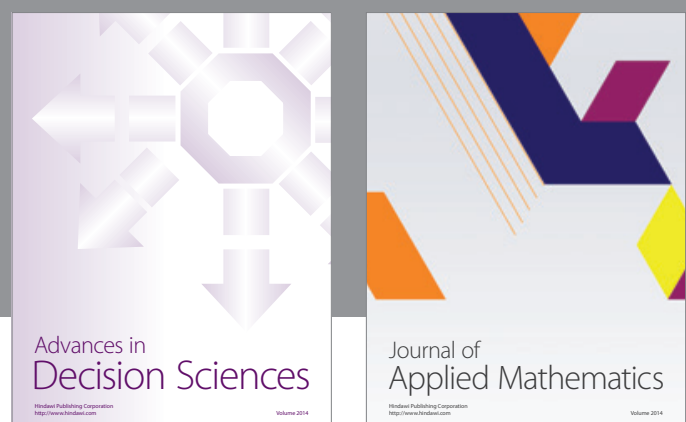

Journal of

Applied Mathematics
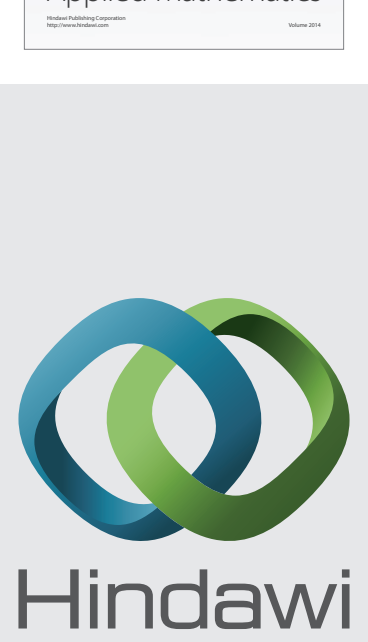

Submit your manuscripts at http://www.hindawi.com
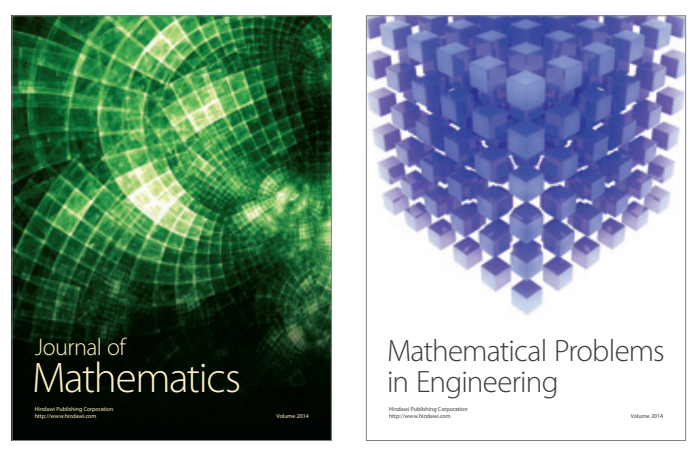

Mathematical Problems in Engineering
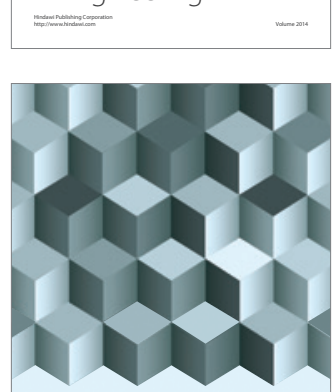

Journal of

Function Spaces
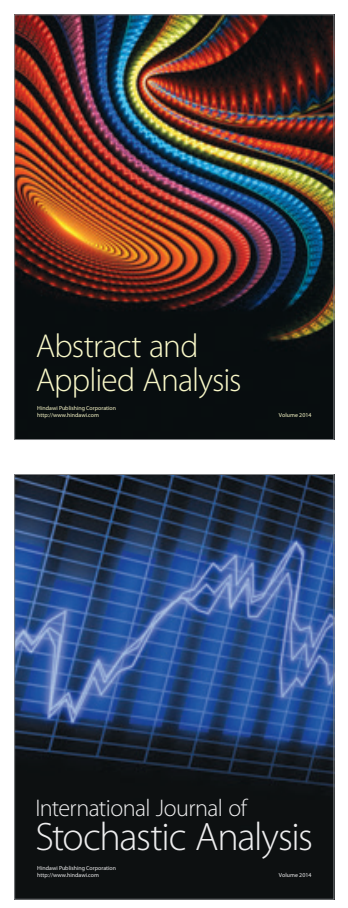

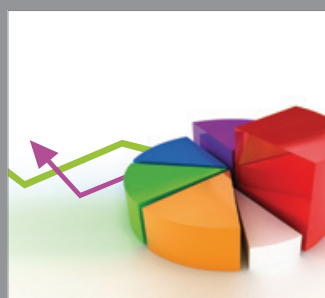

ournal of

Probability and Statistics

Promensencen
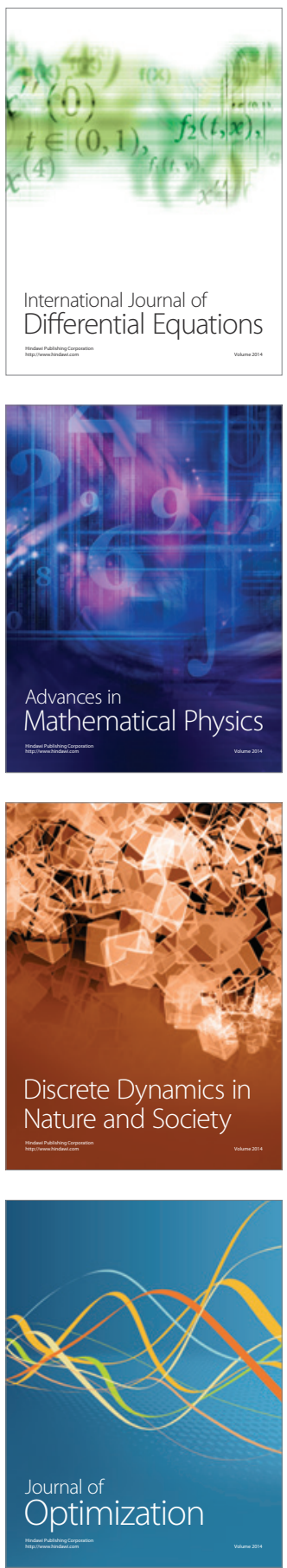